J. DifFERENTIAL GEOMETRY

50 (1998) 417-469

\title{
HAMILTONIAN LOOP GROUP ACTIONS AND VERLINDE FACTORIZATION
}

\author{
E. MEINRENKEN \& C. WOODWARD
}

\begin{abstract}
We prove a formula for $\operatorname{Spin}_{c}$ quantizations of reductions of Hamiltonian actions of loop groups. This includes as a special case the factorization formula for the $\operatorname{Spin}_{c}$ quantization of the moduli space of flat connections over a compact oriented two-manifold.
\end{abstract}

\section{Introduction}

The geometric quantization of the moduli space of flat connections over a surface has been the subject of intensive study from a number of different points of view. Much of the recent work in mathematics has focused on proving formulas discovered by the physicist E. Verlinde [51] in the context of conformal field theory. The two ingredients in Verlinde's approach are the "factorization property", which describes the behavior of the quantization when boundary circles are glued together, and the "fusion rules", which describe the quantization of the moduli space of a three-holed sphere (pair of pants) with boundary components marked by irreducible representations of the loop group.

In the Kähler approach to geometric quantization, one takes the quantization to be the space of sections of a pre-quantum line bundle that are holomorphic with respect to some Kähler structure. In this setting both parts of Verlinde's approach were carried out rigorously by Tsuchiya-Ueno-Yamada [48], using degeneration of a chosen conformal structure on the surface. For further information on this approach,

Received November 14, 1996. 
see Beauville-Laszlo [7], Faltings [14], Kumar-Narasimhan-Ramanathan [30], and Teleman [46] [47] and the references cited therein.

A second definition of geometric quantization uses the index of the

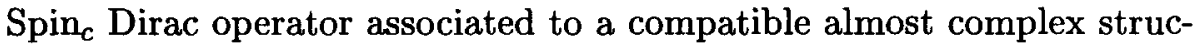
ture and pre-quantum line bundle, which in the presence of a Kähler structure equals the Euler characteristic of the line bundle. This definition works for any pre-quantized symplectic manifold, but it merely gives a number rather than an explicit vector space.

In this paper we give a rigorous proof of the factorization theorem for $\operatorname{Spin}_{c}$ quantization in the context of Hamiltonian loop group actions, along the lines of a strategy outlined by Segal [41] which uses cutting rather than degeneration of the surface. The main point of our approach is that the factorization theorem is a corollary of a "quantization commutes with reduction" theorem for Hamiltonian loop group actions with proper moment maps. The technical fact which allows us to prove a theorem in this generality is the existence of finite-dimensional crosssections for these actions (which are generalizations of the extended moduli spaces of Jeffrey and Huebschmann), by which we manage to avoid any infinite-dimensional analysis. On the down side, the lack of any natural Kähler structures on the cross-sections forces us to work with $\operatorname{Spin}_{c}$ rather than Kähler quantization. The proof eventually reduces to the finite-dimensional version of "quantization commutes with reduction" for $\operatorname{Spin}_{c}$ quantization, which has been proved in general in Meinrenken, Meinrenken-Sjamaar [36],[37].

The broader goal of the paper is to develop the theory of Hamiltonian actions of loop groups with proper moment maps, and to show that many of the properties of Hamiltonian actions of compact groups have loop group generalizations. In particular, we prove a convexity theorem for the image of the moment map.

The reader should be aware that our $\mathrm{Spin}_{c}$ factorization theorem does not quite imply factorization for Kähler quantization, for two reasons. First, the higher cohomology of the pre-quantum line bundle over the moduli spaces is not known to vanish. Vanishing results in the rank-two case are given in Mehta and Ramadas [34] and in the case without markings by Kumar and Narasimhan [29]. Second, in order to define the $\operatorname{Spin}_{c}$ quantization of singular symplectic quotients we use a desingularization procedure which is not known to be Kähler.

The Verlinde formula itself is discussed in Beauville [6] and Szenes [45], and in the survey Sorger [44]. There is an alternative approach to the Verlinde formula based not on the factorization property but rather 
the cohomology ring of the moduli space; see Szenes [45], Jeffrey-Kirwan [24] and Bismut-Labourie [9].

Acknowledgments. We thank S. Chang, L. Jeffrey, S. Martin, A. Szenes, C. Teleman, and M. Thaddeus for helpful discussions. E. M. was supported by a Feodor-Lynen fellowship from the Humboldt Foundation. C. W. was supported by an NSF Postdoctoral Fellowship.

\section{Factorization and quantization commutes with reduction}

In this section we explain how the Verlinde factorization property may be viewed as a manifestation of the "quantization commutes with reduction" principle. The basic idea was outlined by Segal [41].

Let $G$ be a connected and simply-connected compact Lie group and $\Sigma$ a compact, oriented 2-manifold with $b$ boundary components. View the space of Lie-algebra valued 1-forms

$$
\mathcal{A}(\Sigma) \cong \Omega^{1}(\Sigma, \mathfrak{g})
$$

as the space of connections on the trivial bundle $\Sigma \times G$, and let $F_{A} \in$ $\Omega^{2}(\Sigma, \mathfrak{g})$ be the curvature of $A \in \mathcal{A}(\Sigma)$. The set of flat connections

$$
\mathcal{A}_{F}(\Sigma)=\left\{A \in \mathcal{A}(\Sigma) \mid F_{A}=0\right\}
$$

is invariant under the action of the gauge group $\mathcal{G}(\Sigma)=\operatorname{Map}(G, \Sigma)$. Let $\mathcal{G}_{\partial}(\Sigma) \subset \mathcal{G}(\Sigma)$ be the kernel of the restriction map $\mathcal{G}(\Sigma) \rightarrow \mathcal{G}(\partial \Sigma)$, and define

$$
\mathcal{M}(\Sigma):=\mathcal{A}_{F}(\Sigma) / \mathcal{G}_{\partial}(\Sigma)
$$

According to Atiyah and Bott [4] if $\partial \Sigma=\emptyset$ then $\mathcal{M}(\Sigma)$ is a compact, finite-dimensional symplectic space, in general singular. On the other hand if $\partial \Sigma \neq \emptyset$ then $\mathcal{M}(\Sigma)$ is a smooth infinite-dimensional symplectic manifold (see Donaldson [12] and Theorem 3.1 below - details regarding the appropriate choice of Sobolev classes will be given in the following section). It has a residual Hamiltonian action of the gauge group $\mathcal{G}(\partial \Sigma)$ of the boundary with moment map given by minus the restriction to the boundary:

$$
\mathcal{M}(\Sigma) \rightarrow \Omega^{1}(\partial \Sigma, \mathfrak{g}), \quad[A] \mapsto-\iota_{\partial \Sigma}^{*} A .
$$

There is a natural pre-quantum line bundle $L(\Sigma) \rightarrow M(\Sigma)$ together with a pre-quantum lift of a central extension $\widehat{\mathcal{G}(\partial \Sigma)}$ of $\mathcal{G}(\partial \Sigma)$. We 
denote by $L^{m}(\Sigma)$ the $m$-th tensor power of $L(\Sigma)$, and by $\mathcal{M}^{m}(\Sigma)$ the moduli space with $m$ times the equivariant symplectic form, so that $L^{m}(\Sigma) \rightarrow \mathcal{M}^{m}(\Sigma)$ is a pre-quantum bundle.

Let $\Sigma$ be a compact, oriented 2-manifold obtained from a second (possibly disconnected) 2-manifold $\hat{\Sigma}$ by gluing along two boundary components $B_{ \pm} \subset \partial \hat{\Sigma}$. Let $C \subset \Sigma$ be the image of $B_{ \pm}$under the gluing map, and

$$
\mathcal{G}(C) \rightarrow \mathcal{G}\left(B_{+}\right) \times \mathcal{G}\left(B_{-}\right) \subset \mathcal{G}(\partial \hat{\Sigma})
$$

denote the diagonal embedding induced by the gluing. The induced action of $\mathcal{G}(C)$ on $\mathcal{M}(\hat{\Sigma})$ lifts to an action on the pre-quantum bundle $L(\hat{\Sigma}) \rightarrow \mathcal{M}(\hat{\Sigma})$. By Theorem 3.5 below, the moduli space $\mathcal{M}(\Sigma)$ and line bundle $L(\Sigma)$ are given by symplectic reduction by the diagonal action:

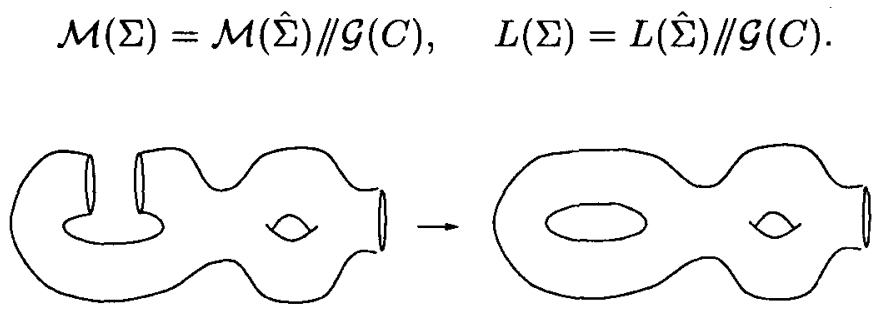

Figure 1. The gluing of $\hat{\Sigma}$ to obtain $\Sigma$

\section{$2.1 \quad$ Factorization}

Let $G$ be a compact Lie group and $M$ a Hamiltonian $G$-space. Suppose that by some quantization procedure one can construct out of these data a virtual representation $Q(M)$ of $G$. The "quantization commutes with reduction" principle (as formulated by Guillemin-Sternberg [18]) says that in this case the quantization of the symplectic reduction $M / / G$ should equal the invariant part of the quantization:

$$
Q(M / / G)=Q(M)^{G}
$$

More generally, if $H$ is another Lie group, and the $G$-action extends to a quantizable Hamiltonian $G \times H$-action, then (2) should hold as an equality of $H$-representations. 
In the context of Kähler quantization (2) was proved in GuilleminSternberg [18] for smooth symplectic quotients and in Sjamaar [42] for singular quotients. For smooth quotients of projectively embedded varieties it follows from the equivalence of geometric invariant theory and symplectic quotients proved by Kempf-Ness and Kirwan; see [26, page 109] and [13, Chapter 6]. For $\mathrm{Spin}_{c}$ quantization, the principle has been proved in Guillemin [17], Meinrenken [35] and Vergne [49] in the abelian case and in Meinrenken [36] for the non-abelian case. The case of singular quotients has been dealt with in Meinrenken-Sjamaar [37].

Heuristically, the factorization property for moduli spaces of flat connections follows from an application of (2) in the setting of Hamiltonian actions of loop groups. Let $\hat{\Sigma}$ be a compact oriented 2-manifold (possibly disconnected), and $\Sigma$ the 2-manifold formed by gluing along two boundary components $B_{ \pm} \subset \partial \hat{\Sigma}$. The gauge group $\mathcal{G}\left(B_{ \pm}\right)$is isomorphic to the loop group

$$
L G=\operatorname{Map}\left(S^{1}, G\right)
$$

of $G$. Equations (1) and (2) would imply an isomorphism of $\widehat{\mathcal{G ( \partial \Sigma )}}$ representations

$$
Q\left(\mathcal{M}^{m}(\Sigma)\right)=Q\left(\mathcal{M}^{m}(\hat{\Sigma})\right)^{L G} \text { (Factorization). }
$$

We want to emphasize that we do not prove the factorization theorem in this form, which would require the construction of the quantization of an infinite dimensional symplectic manifold. Rather, note the following two corollaries of the principle (2) for compact groups $G$. For any dominant weight $\mu$, let $* \mu$ be the dominant weight for the dual representation $V_{\mu}^{*}$, which by Borel-Weil can be realized as the quantization of the coadjoint orbit $\mathcal{O}_{* \mu}=G \cdot * \mu$. The symplectic reduction

$$
M_{\mu}:=M \times \mathcal{O}_{* \mu} / / G
$$

is called the reduction of $M$ at level $\mu$. As a corollary of the principle (2) one has that

$$
Q\left(M_{\mu}\right)=\left(Q(M) \otimes V_{\mu}^{*}\right)^{G}
$$

so that

$$
Q(M)=\oplus_{\mu} Q\left(M_{\mu}\right) V_{\mu} .
$$

Now suppose that $M$ is a compact quantizable Hamiltonian $G \times G$-space, and let $G$ act on $M$ by the diagonal action. Then

$$
Q(M / / G)=Q(M)^{G}=\bigoplus_{\mu, \nu} Q\left(M_{\mu, \nu}\right)\left(V_{\mu} \otimes V_{\nu}\right)^{G}=\bigoplus_{\mu} Q\left(M_{\mu, * \mu}\right)
$$


where the sum is over all dominant weights of $G$.

Our main result is the analogue of this formula for Hamiltonian $L G$ actions with proper moment maps. We denote $\operatorname{Spin}_{c}$ quantization by the symbol RR (for Riemann-Roch number). Let $\Lambda_{m}^{*}$ denote the set of dominant weights at level $m$ (see Section 4.1).

Theorem 2.1 (Factorization Principle). Let $G$ be a compact connected simply-connected Lie group. Let $M$ be a Hamiltonian $L(G \times G)$ Banach manifold with proper moment map at level $m \in \mathbb{N}$ and $\widehat{L G^{2}}$ equivariant pre-quantum line bundle. Then the Riemann-Roch number of $M / / L G$ is

$$
\mathrm{RR}(M / / L G)=\sum_{\mu \in \Lambda_{m}^{*}} \mathrm{RR}\left(M_{\mu, * \mu}\right)
$$

The properness assumption guarantees that all quotients are finite dimensional and compact. Their Riemann-Roch numbers can be defined using desingularization if necessary (see Section 5.3).

For any 2-manifold $\Sigma$ with $b$ boundary components and dominant weight $\nu=\left(\nu_{1}, \ldots, \nu_{b}\right)$ at level $m$, the reduced space $\mathcal{M}^{m}(\Sigma)_{\nu}$ is the moduli space of flat connections with holonomy around the $j$-th boundary component in the conjugacy class of $\exp \left(\nu_{j} / m\right)$. As a special case Theorem 2.1 gives

Theorem 2.2 (Verlinde Factorization). Let $\hat{\Sigma}$ be a compact oriented 2-manifold (possibly disconnected) with $b \geq 2$ boundary components and $\Sigma$ the 2-manifold formed by gluing along two boundary components $B_{ \pm} \subset \partial \hat{\Sigma}$. Given a level $m \in \mathbb{N}$ and dominant weights $\nu=\left(\nu_{1}, \ldots, \nu_{b-2}\right)$ at level $m$, one has

$$
\mathrm{RR}\left(\mathcal{M}^{m}(\Sigma)_{\nu}\right)=\sum_{\mu \in \Lambda_{m}^{*}} \mathrm{RR}\left(\mathcal{M}^{m}(\hat{\Sigma})_{\nu, \mu, * \mu}\right)
$$

\subsection{Fusion product}

We mention briefly two well-known applications of the factorization property. For $\Sigma$ the three-holed sphere the factorization property may be viewed as the associativity of a certain product operation, known as fusion, or Verlinde, product. For some fixed level $m \in \mathbb{Z}_{+}$, the coefficients of the level $m$ fusion product are given by

$$
N_{\mu, \nu ; \alpha}^{m}:=\operatorname{RR}\left(\mathcal{M}^{m}(\Sigma)_{\mu, \nu, * \alpha}\right)
$$

for $\mu, \nu, \alpha \in \Lambda_{m}^{*}$. We claim that coefficients $N_{\mu, \nu, \alpha}^{m}$ are a set of fusion rules, which means that they satisfy the axioms 
- [Commutativity] $N_{\mu, \nu ; \alpha}^{m}=N_{\nu, \mu ; \alpha}^{m}$,

- [Duality] $N_{\mu, \nu ; \alpha}^{m}=N_{* \mu, * \nu ; * \alpha}^{m}$,

- [Identity Element] $N_{\mu, * \nu ; 0}^{m}=N_{\mu, 0 ; \nu}^{m}=\delta_{\mu, \nu}$ and

- [Associativity]

(4)

$$
\sum_{\alpha \in \Lambda_{m}^{*}} N_{\nu, \alpha ; \mu}^{m} N_{\beta, \rho ; \alpha}^{m}=\sum_{\alpha \in \Lambda_{m}^{*}} N_{\beta, \alpha ; \mu}^{m} N_{\nu, \rho ; \alpha}^{m} .
$$

Associativity follows from Theorem 2.2, which implies that both sides of (4) equal

$$
\operatorname{RR}\left(\mathcal{M}^{m}(\tilde{\Sigma})_{* \mu, \nu, \beta, \rho}\right)
$$

where $\tilde{\Sigma}$ is the four-holed sphere. The proof of the other properties is left to the reader.

The structure coefficients (3) define a product operation on

$$
\operatorname{Rep}_{m}(L G)=\mathbb{Z}\left[\Lambda_{m}^{*}\right]
$$

which is called the Verlinde (fusion) ring at level $m$.

\subsection{The Verlinde formula}

As noted by Verlinde, in case $b=0$ and $g \geq 2$ factorization leads to an expression for the Riemann-Roch number of $\mathcal{M}(\Sigma)$ in terms of the symmetric matrix

$$
A_{\beta}^{\alpha}=\operatorname{RR}\left(\mathcal{M}^{m}\left(\Sigma_{1}^{2}\right)_{\alpha, * \beta}\right)=\sum_{\mu, \nu \in \Lambda_{m}^{*}} N_{\mu, \nu ; \alpha}^{m} N_{\mu, \nu ; \beta}^{m} .
$$

In this case, $\Sigma$ can be obtained by gluing together $g-1$ copies of the two-punctured torus $\Sigma_{1}^{2}$, and Theorem 2.1 implies that

$$
\operatorname{RR}\left(\mathcal{M}^{m}(\Sigma)\right)=\operatorname{tr}\left(A^{g-1}\right) .
$$

For information on how to obtain the explicit Verlinde formula from this approach, see Beauville [6].

\section{Moduli spaces of flat connections}

In this section we review the construction of the moduli space $\mathcal{M}(\Sigma)$ and the pre-quantum bundle $L(\Sigma)$ for a surface $\Sigma$ with boundary, and 
give a proof of the "gluing equals reduction" principle. Throughout this section we fix a real number $s>1$ and denote, for any manifold $X$ (possibly with boundary), by $\Omega^{i}(X, \mathfrak{g})$ the space of $\mathfrak{g}$-valued 1-forms of Sobolev class $s-i+\operatorname{dim} X / 2$.

\subsection{Gauge-theoretic construction}

Let $\Sigma$ be an oriented compact 2 -manifold with $b$ boundary components. If $\Sigma$ is connected and has genus $g$, we will write $\Sigma=\Sigma_{g}^{b}$. We denote by $\iota: \partial \Sigma \hookrightarrow \Sigma$ the inclusion of the boundary.

Let $G$ be a connected and simply-connected compact Lie group. We fix an invariant inner product

$$
\operatorname{Tr}: \mathfrak{g} \otimes \mathfrak{g} \rightarrow \mathbb{R}
$$

on the Lie algebra $\mathfrak{g}$, normalized by the requirement that every long root has length $\sqrt{2}$. The inner product induces identifications $\mathfrak{g} \cong \mathfrak{g}^{*}$ and $\mathfrak{t} \cong \mathfrak{t}^{*}$. For $G=S U(n)$, the inner product is given by

$$
\operatorname{Tr}(\xi, \eta)=\frac{-1}{4 \pi^{2}} \operatorname{tr}(\xi \cdot \eta)
$$

Let $\mathcal{A}(\Sigma) \cong \Omega^{1}(\Sigma, \mathfrak{g})$ be the Banach spaces of principal connections on $\Sigma \times G$. It has a symplectic 2 -form given by ${ }^{1}$

$$
\omega_{A}\left(a_{1}, a_{2}\right)=\int_{\Sigma} \operatorname{Tr}\left(a_{1} \wedge a_{2}\right)
$$

For all $A \in \mathcal{A}(\Sigma)$, we denote by

$$
\mathrm{d}_{A}=\mathrm{d}+[A, \cdot]: \Omega^{i}(\Sigma, \mathfrak{g}) \rightarrow \Omega^{i+1}(\Sigma, \mathfrak{g})
$$

the associated covariant derivative, and by

$$
F_{A}=\mathrm{d} A+\frac{1}{2}[A, A] \in \Omega^{2}(\Sigma, \mathfrak{g})
$$

its curvature.

Let $\mathcal{G}(\Sigma)=\operatorname{Map}(\Sigma, G)$ be the group of gauge transformations of Sobolev class $s+1$. Since $s+1>\operatorname{dim}(\Sigma) / 2$, the group $\mathcal{G}(\Sigma)$ consists of

\footnotetext{
${ }^{1}$ By a symplectic structure on a Banach manifold $M$ we mean a closed 2-form $\omega$ that is weakly non-degenerate, that is, for any $m \in M$ the map $T_{m} M \rightarrow T_{m}^{*} M$ induced by $\omega$ is injective (see e.g. [1]).
} 
continuous maps, and is a Banach Lie group with Lie algebra $\Omega^{0}(\Sigma, \mathfrak{g})$. The action of $\mathcal{G}(\Sigma)$ on $\Sigma \times G$ induces an action on $\mathcal{A}(\Sigma)$ given by

$$
g \cdot A=\operatorname{Ad}_{g}(A)-\mathrm{d} g g^{-1} .
$$

This action preserves the symplectic structure, and the fundamental vector field corresponding to $\xi \in \Omega^{0}(\Sigma, \mathfrak{g})$ is given by

$$
\xi_{\mathcal{A}(\Sigma)}(A)=-\mathrm{d}_{A}(\xi)
$$

Recall that for any manifold $X$ with boundary, the restriction map $C^{\infty}(X) \rightarrow C^{\infty}(\partial X)$ to the boundary extends for $r>\frac{\operatorname{dim}(X)}{2}$ to a continuous surjection of Sobolev spaces

$$
H_{(r)}(X) \rightarrow H_{\left(r-\frac{1}{2}\right)}(\partial X)
$$

(see e.g. [10, Chapter 11]). Hence there is a surjective map from $\mathcal{A}(\Sigma)$ to the space $\mathcal{A}(\partial \Sigma) \cong \Omega^{1}(\partial \Sigma, \mathfrak{g})$ of connections over $\partial \Sigma$. According to Atiyah and Bott [4], [3], a moment map $\Phi$ for the action of $\mathcal{G}(\Sigma)$ is given by

$$
\Phi: \mathcal{A}(\Sigma) \rightarrow \Omega^{2}(\Sigma, \mathfrak{g}) \oplus \Omega^{1}(\partial \Sigma, \mathfrak{g}), A \mapsto\left(F_{A},-\iota^{*} A\right) .
$$

More precisely, we have

$$
\iota\left(\xi_{\mathcal{A}(\Sigma)}\right) \omega=d\langle\Phi, \xi\rangle
$$

where

$$
\langle\Phi(A), \xi\rangle=\int_{\Sigma} \operatorname{Tr}\left(F_{A} \xi\right)-\int_{\partial \Sigma} \iota^{*} \operatorname{Tr}(A \xi)
$$

Let $\mathcal{G}_{\partial}(\Sigma) \subset \mathcal{G}(\Sigma)$ be the kernel of the restriction map to the boundary so that there is an exact sequence

$$
1 \rightarrow \mathcal{G}_{\partial}(\Sigma) \rightarrow \mathcal{G}(\Sigma) \rightarrow \mathcal{G}(\partial \Sigma) \rightarrow 1
$$

The moment map for the action of $\mathcal{G}_{\partial}(\Sigma)$ on $\mathcal{A}(\Sigma)$ is $A \mapsto F_{A}$, and hence the symplectic quotient of $\mathcal{A}(\Sigma)$ by $\mathcal{G}_{\partial}(\Sigma)$ is

$$
\mathcal{M}(\Sigma):=\mathcal{A}_{F}(\Sigma) / \mathcal{G}_{\partial}(\Sigma),
$$

where $\mathcal{A}_{F}(\Sigma) \subset \mathcal{A}(\Sigma)$ is the space of flat connections.

\section{Theorem 3.1.}

a. If $\partial \Sigma=\emptyset$ then $\mathcal{M}(\Sigma)$ is a compact, finite dimensional stratified symplectic space (in general singular). 
b. If $\partial \Sigma \neq \emptyset$ then $\mathcal{M}(\Sigma)$ is a smooth infinite-dimensional symplectic manifold. It has a residual Hamiltonian action of the gauge group $\mathcal{G}(\partial \Sigma)$ of the boundary with moment map

$$
\Phi: \mathcal{M}(\Sigma) \rightarrow \Omega^{1}(\partial \Sigma, \mathfrak{g}), \quad[A] \mapsto-\iota^{*} A
$$

The first assertion is due to Atiyah-Bott [4], at least in the case that the quotient is smooth. The second assertion is proved in Donaldson [12]. The idea of proof is as follows. An atlas for $\mathcal{M}(\Sigma)$ for $b>0$ is constructed from local slices for the gauge group action as in [12], [13], [4]. Fix a Riemannian metric on $\Sigma$, and let $A \in \mathcal{A}(\Sigma)$ be an irreducible connection. By the implicit function theorem, any connection $A+a$, with $a$ small, can be gauge transformed by a unique element $g \in \mathcal{G}_{\partial}(\Sigma)$ into Coulomb gauge with respect to $A$, that is, so that

$$
d_{A}^{*}(g \cdot(A+a)-A)=0 .
$$

In other words, a neighborhood of $A$ in $A+\operatorname{ker}\left(\mathrm{d}_{A}^{*}\right)$ is a slice for the $\mathcal{G}_{\partial}(\Sigma)$-action on $\mathcal{A}(\Sigma)$. For any $A \in \mathcal{A}_{F}(\Sigma)$ one defines a local moduli space to be a neighborhood of $A$ in $\mathcal{A}_{F}(\Sigma)$ of the form

$$
V_{A} \subset\left\{A+a \in \Omega^{1}(\Sigma, \mathfrak{g}) \mid F_{A+a}=0, d_{A}^{*} a=0\right\} .
$$

Using the implicit function theorem again, one shows that if $V_{A}$ is taken sufficiently small, $V_{A} \subset \mathcal{A}_{F}(\Sigma)$ is a smooth Banach submanifold locally homeomorphic to its tangent space at 0

$$
T_{0}\left(V_{A}\right)=\left\{a \in \Omega^{1}(\Sigma, \mathfrak{g}) \mid \mathrm{d}_{A} a=0, \mathrm{~d}_{A}^{*} a=0\right\} .
$$

The sets $V_{A}$ together with the coordinate mappings $V_{A} \rightarrow T_{0}\left(V_{A}\right)$ give an atlas for $\mathcal{M}(\Sigma)$. The Hodge *-operator induced from the choice of metric on $\Sigma$ defines a complex structure on $T_{A}(\mathcal{A}(\Sigma))$ which is compatible with the symplectic structure. The tangent space $T_{0}\left(V_{A}\right)$ is a complex (and therefore symplectic) subspace which implies that $\mathcal{M}(\Sigma)$ is a Banach Kähler manifold. In this paper we will not make use of the complex structure.

\subsection{Holonomy description}

Let $S^{1}=\mathbb{R} / \mathbb{Z}$ be the circle and $L G$ the loop group of $G$, defined as the Banach Lie group consisting of maps $S^{1} \rightarrow G$ of Sobolev class $s+\frac{1}{2}$, 
with Lie algebra $L \mathfrak{g}=\Omega^{0}\left(S^{1}, \mathfrak{g}\right)$. We define $L \mathfrak{g}^{*}:=\Omega^{1}\left(S^{1}, \mathfrak{g}\right)$, viewed as the affine space of connections on $S^{1}$. We take the action of $L G$ on $L \mathfrak{g}^{*}$ to be the gauge action $g \cdot \xi=\operatorname{Ad}_{g} \xi-\mathrm{d} g g^{-1}$. The natural pairing of $L \mathfrak{g}^{*}$ with $L \mathfrak{g}$ given by integration and inner product on $\mathfrak{g}$ makes $L \mathfrak{g}^{*}$ into a subset of the topological dual of $L \mathfrak{g}$. We denote by

$$
\text { Hol : } L \mathfrak{g}^{*} \rightarrow G
$$

the smooth map that sends $\eta \in L \mathfrak{g}^{*}$ to the holonomy of $\eta$ around $S^{1}$. The map Hol has the equivariance property $\operatorname{Hol}(g \cdot \xi)=\operatorname{Ad}_{g(0)} \operatorname{Hol}(\xi)$. The Lie algebra $\mathfrak{g}$ embeds as the space of constant one-forms

$$
\mathfrak{g} \rightarrow L \mathfrak{g}^{*}, \quad \zeta \mapsto \zeta d t .
$$

The restriction of Hol to $\mathfrak{g} \subset L \mathfrak{g}^{*}$ is given by $\operatorname{Hol}(\zeta)=\exp (-\zeta)$.

The moduli space $\mathcal{M}(\Sigma)$ can be described in terms of parallel transports as follows. A choice of parametrization $\partial \Sigma \cong\left(S^{1}\right)^{b}$ induces an isomorphism

$$
\mathcal{G}(\partial \Sigma) \cong L G^{b}
$$

We take the parametrization to be orientation reversing, changing the sign in (5) so that the moment map becomes an equivariant map $\mathcal{M}(\Sigma) \rightarrow$ $\left(L \mathfrak{g}^{*}\right)^{b}$.

Theorem 3.2. If $b \geq 1$, the moduli space $\mathcal{M}(\Sigma)$ is isomorphic to the set of $(a, c, \xi) \in G^{2 g} \times G^{b-1} \times\left(L \mathfrak{g}^{*}\right)^{b}$ such that

$$
\prod_{i=1}^{2 g}\left[a_{2 i-1}, a_{2 i}\right]=\prod_{i=1}^{b} \operatorname{Ad}_{c_{i}} \operatorname{Hol}\left(\xi_{i}\right)
$$

where $c_{1}=1$. This is a smooth submanifold of $G^{2 g} \times G^{b-1} \times\left(L \mathfrak{g}^{*}\right)^{b}$, and the identification with $\mathcal{M}(\Sigma)$ is an $L G^{b}$-equivariant diffeomorphism. Here the action of $g=\left(g_{1}, \ldots, g_{b}\right) \in L G^{b}$ on $G^{2 g} \times G^{b-1} \times\left(L \mathfrak{g}^{*}\right)^{b}$ is given by

$g \cdot a_{i}=\operatorname{Ad}_{g_{1}(0)} a_{i}, \quad g \cdot c_{j}=g_{1}(0) c_{j} g_{j}(0)^{-1}, \quad g \cdot \xi_{j}=\operatorname{Ad}_{g_{j}} \cdot \xi_{j}-d g_{j} g_{j}^{-1}$

and the moment map is given by projection to the $\left(L \mathrm{~g}^{*}\right)^{b}$-factor.

Proof. The diffeomorphism is given as follows. Let $\iota_{j}: S^{1} \rightarrow \partial \Sigma \hookrightarrow$ $\Sigma$ denote orientation-reversing parametrizations of the boundary circles, and let $B_{j}=\iota_{j}\left(S^{1}\right)$. This gives identifications $\mathcal{G}(\partial \Sigma) \cong L G^{b}$ and $\Omega^{1}(\partial \Sigma, \mathfrak{g}) \cong\left(L \mathfrak{g}^{*}\right)^{b}$ and base points $x_{j}=\iota_{j}(1) \in B_{j}$. Now let $\rho_{1}, \ldots, \rho_{2 g}$ 
be smooth loops based at $x_{1}$, and $C_{2}, \ldots, C_{b}$ smooth paths from $x_{1}$ to $x_{2}, \ldots, x_{n}$, in such a way that $\pi_{1}(\Sigma)$ is generated by the $\rho_{i}$ together with $B_{1}$ and $C_{j}^{-1} B_{j} C_{j}$, subject to the relation

$$
\prod_{i=1}^{g}\left[\rho_{2 i-1}, \rho_{2 i}\right]=B_{1}\left(C_{2}^{-1} B_{2} C_{2}\right) \ldots\left(C_{b}^{-1} B_{b} C_{b}\right) .
$$

Consider the map

$$
\hat{f}: \mathcal{A}_{F}(\Sigma) \rightarrow G^{2 g} \times G^{b-1} \times\left(L \mathfrak{g}^{*}\right)^{b}
$$

that takes any flat connection $A$ to $(a, c, \xi)$ where $a_{i}$ is the holonomy around $\rho_{i}, c_{j}$ the parallel transport along $C_{j}$, and $\xi_{j}=\iota_{j}^{*} A$. Then

$$
\prod_{i=1}^{g}\left[a_{2 i-1}, a_{2 i}\right]=\prod_{j=1}^{b} \operatorname{Ad}_{c_{j}}\left(\operatorname{Hol}\left(\xi_{j}\right)\right)
$$

where we set $c_{1}:=1$. The map $\hat{f}$ is equivariant with respect to the action of the gauge group $\mathcal{G}(\Sigma)$ given on $G^{2 g} \times G^{b-1} \times\left(L \mathfrak{g}^{*}\right)^{b}$ by

$$
g \cdot a_{i}=\operatorname{Ad}_{g\left(x_{1}\right)} a_{i}, \quad g \cdot c_{j}=g\left(x_{1}\right) c_{j} g\left(x_{j}\right)^{-1}, \quad g \cdot \xi_{j}=\left(g \mid B_{j}\right) \cdot \xi_{j} .
$$

Moreover, $\hat{f}$ is surjective onto the set of all $(a, c, \xi)$ satisfying (8): First, as in the case without boundary, one can construct a smooth connection that has the required holonomies $a_{i}$ and $\operatorname{Ad}_{c_{j}}\left(\mathrm{Hol}\left(\xi_{j}\right)\right)$. Secondly, one can act by an element $g$ of $\mathcal{G}(\Sigma)$ with $g\left(x_{1}\right)=1$ (which does not change the holonomies) to obtain the required values of $\xi_{j}$. This gives the correct values for the parallel transport $c_{j}$ along the curves $C_{j}$, up to an action of the centralizer of $\operatorname{Hol}\left(\xi_{j}\right)$ from the right. Now evaluation at $x_{j}$ gives an isomorphism of $G_{\mathrm{Hol}\left(\xi_{j}\right)}$ with the stabilizer $(L G)_{\xi_{j}}$. Thus finally we can act by an element $g^{\prime} \in \mathcal{G}(\Sigma)$ with $g^{\prime}\left(x_{1}\right)=1$ and $g^{\prime}\left(x_{j}\right) \in G_{\mathrm{Hol}\left(\xi_{j}\right)}$ for $j \geq 2$ to obtain the correct values of $c_{j}$.

We next show that the fiber of $\hat{f}$ over $(a, c, \xi)$ is equal to $\mathcal{G}_{\partial}(\Sigma)$. To see this, one may assume after acting by $\mathcal{G}(\Sigma)$ that $\xi$ is smooth. Let $A_{1}, A_{2} \in \hat{f}^{-1}(a, c, \xi)$. For $x \in \Sigma$, choose a smooth path $\tau$ from $x_{1}$ to $x$, and let $g(x) \in G$ defined by parallel transport along $\tau$ by $A_{1}$, followed by parallel transport along $\tau^{-1}$ by $A_{2}$. This is independent of the choice of $\tau$, and therefore gives a smooth function $g: \Sigma \rightarrow G$ with $g \in \mathcal{G}_{\partial}(\Sigma)$ and $g \cdot A_{1}=A_{2}$. By a similar argument, one shows that the kernel of 
the tangent map to $\hat{f}$ is equal to the tangent space to the $\mathcal{G}_{\partial}(\Sigma)$-orbit. Hence $\hat{f}$ descends to a smooth embedding

$$
f: \mathcal{M}(\Sigma) \rightarrow G^{2 g} \times G^{b-1} \times\left(L \mathfrak{g}^{*}\right)^{b}
$$

with image equal to the set (8). q.e.d.

Corollary 3.3. The moment map

$$
\Phi: \mathcal{M}(\Sigma) \rightarrow \Omega^{1}(\partial \Sigma, \mathfrak{g}) \cong\left(L \mathfrak{g}^{*}\right)^{b}
$$

is proper.

Example 3.4. The moduli space $\mathcal{M}\left(\Sigma_{0}^{1}\right)$ for the disk is the space of $\xi \in L \mathfrak{g}^{*}$ such that $\operatorname{Hol}(\xi)=e$, that is, the orbit of $L G$ through $0 \in L \mathfrak{g}^{*}$. Since the stabilizer of the trivial connection is the group $G$ of constant loops, $\mathcal{M}\left(\Sigma_{0}^{1}\right)$ is diffeomorphic to the space $L G / G=\Omega G$ of based loops in $G$ (fundamental homogeneous space of $L G$ ). See for example [39], [16].

\subsection{The pre-quantum line bundle}

Recall that a pre-quantum line bundle $L$ for a symplectic manifold $(M, \omega)$ is a Hermitian line bundle equipped with invariant connection $\nabla$ whose curvature is $-2 \pi i$ times the symplectic form. By Kostant's theorem [28], if $M$ is connected, the group $\operatorname{Aut}_{\nabla}(L)$ of connection preserving Hermitian bundle automorphisms is a central extension by $S^{1}$ of its image in the group $\operatorname{Diff}_{\omega}(M)$ of symplectomorphisms of $M$. Given a connected Lie group $K$ and a symplectic action $K \rightarrow \operatorname{Diff}_{\omega}(M)$ one obtains a central extension

$$
1 \rightarrow S^{1} \rightarrow \widehat{K} \rightarrow K \rightarrow 1,
$$

together with an action $\widehat{K} \rightarrow \operatorname{Aut}_{\nabla}(L)$. If the action of $K$ is in fact Hamiltonian, with equivariant moment map $\Phi: M \rightarrow \mathfrak{k}^{*}$, the Kostant formula

$$
\xi_{L}=\operatorname{Lift}\left(\xi_{M}\right)+\langle\Phi, \xi\rangle \frac{\partial}{\partial t}
$$

(where $\frac{\partial}{\partial t}$ is the generating vector field for the scalar $S^{1}$-action on the fiber) defines a Lie algebra splitting $\hat{\mathfrak{k}} \cong \mathbb{R} \oplus \mathfrak{k}$, or equivalently an invariant flat connection on the bundle (9). If $\pi_{0}(K)=\{1\}$ and if the 
holonomy of this connection is trivial (for example if $\pi_{1}(K)=\{1\}$ ) one obtains in this way a lift $K \rightarrow \operatorname{Aut}_{\nabla}(L)$. One calls the resulting $K$-equivariant line bundle $L$ a pre-quantum line bundle for the Hamiltonian $K$-manifold $(M, \omega, \Phi)$. If $K$ acts freely on the zero level set $\Phi^{-1}(0)$, then the connection $\nabla$ induces a pre-quantum connection on the reduced line bundle

$$
L / / K=\left(L \mid \Phi^{-1}(0)\right) / K .
$$

We now explain following Mickelsson [38], Ramadas-Singer-Weitsman [40], and Witten [52] how to construct a pre-quantum line bundle $L(\Sigma)$ over $\mathcal{M}(\Sigma)$ which carries an action of a central extension of $\mathcal{G}(\partial \Sigma) \cong$ $L G^{b}$. The line bundle $L(\Sigma)$ will be obtained from a pre-quantum line bundle over $\mathcal{A}(\Sigma)$ by reduction.

Since $\mathcal{A}(\Sigma)$ is an affine space, the trivial line bundle $\mathcal{A}(\Sigma) \times \mathbb{C}$ with connection 1-form

$$
\theta_{A}: T_{A} \mathcal{A}(\Sigma) \cong \Omega^{1}(\Sigma, \mathfrak{g}) \rightarrow \mathbb{R}, a \mapsto \frac{1}{2} \int_{\Sigma} \operatorname{Tr}(a \wedge A)
$$

is a pre-quantum line bundle. The central $S^{1}$-extension $\widehat{\mathcal{G}(\Sigma)}$ of the gauge group defined by the cocycle

$$
c\left(g_{1}, g_{2}\right)=\exp \left(-\pi i \int_{\Sigma} \operatorname{Tr}\left(g_{1}^{-1} \mathrm{~d} g_{1} \wedge \mathrm{d} g_{2} g_{2}^{-1}\right)\right),
$$

acts on $\mathcal{A}(\Sigma) \times \mathbb{C}$ by $\theta$-preserving automorphisms via

$$
(g, z) \cdot(A, w)=\left(g \cdot A, \exp \left(\pi i \int_{\Sigma} \operatorname{Tr}\left(g^{-1} \mathrm{~d} g \wedge A\right)\right) z w\right) .
$$

We show that this extension has a canonical trivialization over the subgroup $\mathcal{G}_{\partial}(\Sigma) \subset \mathcal{G}(\Sigma)$ so that $\mathcal{G}_{\partial}(\Sigma)$ acts on $\mathcal{A}(\Sigma) \times \mathbb{C}$. Using the fact that $\pi_{0}(G), \pi_{1}(G), \pi_{2}(G)$ are all trivial, one can show that the gauge group $\mathcal{G}(\Sigma)$ (and also $\mathcal{G}_{\partial}(\Sigma)$ ) is connected:

$$
\pi_{0}(\mathcal{G}(\Sigma))=\pi_{0}\left(\mathcal{G}_{\partial}(\Sigma)\right)=\{1\} .
$$

However, the fundamental groups of $\mathcal{G}(\Sigma), \mathcal{G}_{\partial}(\Sigma)$ are non-trivial in general. On the Lie algebra level, $\operatorname{Lie}(\widehat{\mathcal{G}(\Sigma)})$ is the central extension by $\mathbb{R}$ of $\operatorname{Lie}(\mathcal{G}(\Sigma))$ defined by the cocycle

$$
\left(\xi_{1}, \xi_{2}\right) \mapsto-\int_{\Sigma} \operatorname{Tr}\left(\mathrm{d} \xi_{1} \wedge \mathrm{d} \xi_{2}\right)=-\int_{\partial \Sigma} \operatorname{Tr}\left(\xi_{1} \mathrm{~d} \xi_{2}\right)
$$


that is, $\operatorname{Lie}(\widehat{\mathcal{G}(\Sigma)})=\operatorname{Lie}(\mathcal{G}(\Sigma)) \oplus \mathbb{R}$ with bracket

$$
\left[\left(\xi_{1}, t_{1}\right),\left(\xi_{2}, t_{2}\right)\right]=\left(\left[\xi_{1}, \xi_{2}\right],-\int_{\partial \Sigma} \operatorname{Tr}\left(\xi_{1} \mathrm{~d} \xi_{2}\right)\right) .
$$

One checks that the pre-quantum lift $(10)$ corresponds to the inclusion of $\operatorname{Lie}(\mathcal{G}(\Sigma))$ in $\operatorname{Lie}(\widehat{\mathcal{G}(\Sigma)})$ as the first summand. Notice however that since the moment map for the $\mathcal{G}(\Sigma)$-action is equivariant only over the subgroup $\mathcal{G}_{\partial}(\Sigma)$, the connection on $\widehat{\mathcal{G}(\Sigma)} \rightarrow \mathcal{G}(\Sigma)$ obtained in this way is flat only over $\mathcal{G}_{\partial}(\Sigma)$. We will now check that this flat connection has trivial holonomy, or equivalently that the restriction to $\mathcal{G}_{\partial}(\Sigma)$ of the cocycle $c$ in Equation (11) is a coboundary.

Suppose first that $\partial \Sigma=\emptyset$, so that $\mathcal{G}_{\partial}(\Sigma)=\mathcal{G}(\Sigma)$. Let $X$ be any compact oriented 3-manifold with boundary $\partial X=\Sigma$ and $\hat{g} \in \operatorname{Map}(X, G)$ an extension of $g$ of Sobolev class $s+\frac{3}{2}$. Define

$$
\Gamma: \mathcal{G}(\Sigma) \rightarrow S^{1}, g \mapsto \exp \left(\frac{i \pi}{6} \int_{X} \operatorname{Tr}\left(\hat{g}^{-1} \mathrm{~d} \hat{g} \wedge\left[\hat{g}^{-1} \mathrm{~d} \hat{g}, \hat{g}^{-1} \mathrm{~d} \hat{g}\right]\right)\right) .
$$

The map $\Gamma$ is independent of the choice of $X$ and $\hat{g}$ and satisfies the coboundary property

$$
\Gamma\left(g_{1} g_{2}\right)=\Gamma\left(g_{1}\right) \Gamma\left(g_{2}\right) c\left(g_{1}, g_{2}\right) .
$$

The group homomorphism $\mathcal{G}(\Sigma) \rightarrow \widehat{\mathcal{G}(\Sigma)}, g \mapsto(g, \Gamma(g))$ defines the trivialization.

In the case $\partial \Sigma \neq \emptyset$, consider the 2-manifold $\bar{\Sigma} \supset \Sigma$ without boundary obtained from $\Sigma$ by "capping off" the boundary components. Let $\bar{\Gamma}: \mathcal{G}(\bar{\Sigma}) \rightarrow S^{1}$ be defined as before. Let $\mathcal{G}_{c}(\Sigma) \subset \mathcal{G}(\Sigma)$ be the subgroup of gauge transformations in the kernel of the restriction map $\mathcal{G}(\bar{\Sigma}) \rightarrow \mathcal{G}(\bar{\Sigma}-\Sigma)$. Then the restriction $\Gamma$ of $\bar{\Gamma}$ to $\mathcal{G}_{c}(\Sigma)$ satisfies $(12)$ and therefore shows that the extension is trivial over $\mathcal{G}_{c}(\Sigma)$. Now consider $\mathcal{G}_{\partial}(\Sigma) \supset \mathcal{G}_{c}(\Sigma)$. One sees easily that any loop in $\mathcal{G}_{\partial}(\Sigma)$ can be deformed to a loop in $\mathcal{G}_{c}(\Sigma)$, i.e. that the natural map $\pi_{1}\left(\mathcal{G}_{c}(\Sigma)\right) \rightarrow \pi_{1}\left(\mathcal{G}_{\partial}(\Sigma)\right)$ is surjective. It follows that the central extension is trivial over $\mathcal{G}_{\partial}(\Sigma)$ as well. The quotient

$$
\widehat{\mathcal{G}(\partial \Sigma)}=\widehat{\mathcal{G}(\Sigma)} / \mathcal{G}_{\partial}(\Sigma)
$$

is the (unique) central extension of $\mathcal{G}(\partial \Sigma)$ defined by the Lie algebra cocycle

$$
\left(\xi_{1}, \xi_{2}\right) \mapsto-\int_{\partial \Sigma} \operatorname{Tr}\left(\xi_{1} \mathrm{~d} \xi_{2}\right)
$$


It follows that $\widehat{\mathcal{G (}(\partial \Sigma)}$ is just the basic central extension $\widehat{L G^{b}} \rightarrow L G^{b}$. (In fact, the above construction with $\Sigma$ the 2-disk is precisely Mickelsson's construction [38] of $\widehat{L G}$.)

We define the pre-quantum line bundle $L(\Sigma)$ as the quotient

$$
L(\Sigma)=(\mathcal{A}(\Sigma) \times \mathbb{C}) / / \mathcal{G}_{\partial}(\Sigma)=\left(\mathcal{A}_{F}(\Sigma) \times \mathbb{C}\right) / \mathcal{G}_{\partial}(\Sigma) .
$$

The products $V_{A} \times \mathbb{C} \cong T_{0}\left(V_{A}\right) \times \mathbb{C}$ are slices for the $\mathcal{G}_{\partial}(\Sigma)$-action on $\mathcal{A}_{F}(\Sigma) \times \mathbb{C}$, and give local bundle charts for the line bundle $L(\Sigma)$.

The connection on $\mathcal{A}(\Sigma) \times \mathbb{C}$ descends to a connection on $L(\Sigma)$, so that $L(\Sigma)$ is a pre-quantum line bundle over $\mathcal{M}(\Sigma)$ which carries an action of the central extension $\widehat{\mathcal{G}(\partial \Sigma)}$.

Question. Does the pre-quantum line bundle admit a holonomy description?

\subsection{Gluing equals reduction}

Let $\Sigma$ be a compact oriented 2-manifold obtained from a second (possibly disconnected) 2-manifold $\hat{\Sigma}$ by gluing along two boundary components $B_{ \pm} \subset \partial \hat{\Sigma}$. In this case $\mathcal{M}(\Sigma)$ can be obtained from $\mathcal{M}(\hat{\Sigma})$ by a symplectic reduction. Let $\pi: \hat{\Sigma} \rightarrow \Sigma$ be the gluing map and $C=\pi\left(B_{+}\right)=\pi\left(B_{-}\right)$. The map $\pi$ induces identifications

$$
\mathcal{G}(C) \cong \mathcal{G}\left(B_{ \pm}\right) .
$$

Consider the diagonal embedding

$$
\mathcal{G}(C) \rightarrow \mathcal{G}\left(B_{+}\right) \times \mathcal{G}\left(B_{-}\right) \subset \mathcal{G}(\partial \hat{\Sigma}) .
$$

This embedding lifts to the central extension and induces an action of $\mathcal{G}(C)$ on $L(\hat{\Sigma}) \rightarrow \mathcal{M}(\hat{\Sigma})$. We have the following theorem which we learned from S. Martin:

Theorem 3.5. The moduli space $\mathcal{M}(\Sigma)$ and line bundle $L(\Sigma)$ are given by symplectic reduction by the diagonal action:

$$
\mathcal{M}(\Sigma)=\mathcal{M}(\hat{\Sigma}) / / \mathcal{G}(C), \quad L(\Sigma)=L(\hat{\Sigma}) / / \mathcal{G}(C)
$$

Proof. If the orientation on $C$ is the one induced from that on $B_{-}$, then the moment map for the diagonal action is given by

$$
[\hat{A}] \mapsto \iota_{+}^{*} \hat{A}-\iota_{-}^{*} \hat{A} .
$$


Therefore, if an equivalence class $[\hat{A}]$ is in the zero level set, then the pullbacks of the representative $\hat{A}$ to $B_{ \pm}$are equal. Since flat connections are determined up to gauge equivalence by their holonomies, one can choose a representative $\hat{A}$ which in a collar neighborhood of the boundary is given by a pull-back from $B_{ \pm}$. The two sides patch together to form a connection $A$ on $\Sigma$. Therefore we have a map

$$
\mathcal{M}(\hat{\Sigma}) / / \mathcal{G}(C) \rightarrow \mathcal{M}(\Sigma)
$$

which is easily seen to be bijective.

We now prove that the map is a diffeomorphism. Let $A \in \mathcal{A}_{F}(\Sigma)$ be any fixed connection, and $V_{A} \subset \mathcal{A}_{F}(\Sigma)$ the corresponding local moduli space defined in (7). Define a map

$$
\psi: V_{A} \times \mathcal{G}(C) \rightarrow \mathcal{M}(\hat{\Sigma}),(A+a, h) \mapsto h \cdot\left[\pi^{*}(A+a)\right] .
$$

Notice that this map is $\mathcal{G}(C)$-equivariant and that its image is contained in the zero level set of $\mathcal{M}(\hat{\Sigma})$. To show that $\psi$ is a diffeomorphism from a small neighborhood of $(0, e)$ onto its image, we can assume after acting by a suitable element of $\mathcal{G}(\Sigma)$ that $A$ is smooth. Since the map (13) is a homeomorphism it suffices to prove that $\psi$ is an immersion, that is, the tangent map to $\psi$ at $(0, e)$ is injective and has closed image. In terms of the local moduli space $V_{\pi^{*} A}$, the map $\psi$ is given as

$$
\psi: V_{A} \times \mathcal{G}(C) \rightarrow V_{\pi^{*} A},(A+a, h) \mapsto \tilde{h} \cdot \pi^{*}(A+a),
$$

where $\tilde{h} \in \mathcal{G}(\hat{\Sigma})$ is the unique gauge transformation such that $\tilde{h} \mid B_{ \pm}=h$ and $\tilde{h} \mid\left(\partial \Sigma-\left(B_{+} \cup B_{-}\right)\right)=1$, and such the right-hand side of (14) lies in $V_{\pi^{*} A}$. The tangent map is given by

$$
\mathrm{d}_{(0, e)} \psi(b, \eta)=\pi^{*} b-\mathrm{d}_{\pi^{*} A} \tilde{\eta},
$$

where $\tilde{\eta} \in \Omega^{0}(\hat{\Sigma}, \mathfrak{g})$ is the unique solution of the Dirichlet problem

$$
\mathrm{d}_{\pi^{*} A}^{*} \mathrm{~d}_{\pi^{*} A} \tilde{\eta}=0, \iota_{ \pm}^{*} \tilde{\eta}=\eta|C, \tilde{\eta}|\left(\partial \hat{\Sigma} \backslash\left(B_{+} \cup B_{-}\right)\right)=0 .
$$

Suppose that $(a, \eta) \in \operatorname{ker} d_{(0, e)} \psi$. Since $\tilde{\eta}$ is continuous, and since the restrictions of $\tilde{\eta}$ to $B_{ \pm}$are equal, $\tilde{\eta}=\pi^{*} \zeta$ is the pullback of a continuous function $\zeta \in C^{0}(\Sigma, \mathfrak{g})$. Then $d_{A} \zeta=a$ in the sense of distributions, which by ellipticity of $d_{A}$ on 0 -forms implies that the $\zeta$ is smooth. Since $d_{A}^{*} d_{A} \zeta=0$ and $\zeta \mid \partial \Sigma=0$ it follows that $\zeta=0$ and hence $a=0$, as required. This shows the first claim. The image of $\mathrm{d} \psi$ is closed by ellipticity of $\mathrm{d}_{\pi^{*} A}$. q.e.d. 


\section{Hamiltonian loop group actions}

In the previous section we have shown that the moduli space $\mathcal{M}(\Sigma)$ for a compact oriented 2-manifold with $b>0$ boundary components is a Hamiltonian $L G^{b}$-manifold with proper moment map. In this section we discuss the general theory of Hamiltonian loop group manifolds.

\subsection{Definition of Hamiltonian loop group actions}

Let $G$ be a connected, simply connected compact Lie group, $T$ a maximal torus and $W=N_{G}(T) / T$ the Weyl group. The integral lattice $\{\xi \in \mathfrak{t} \mid \exp (\xi)=1\}$ will be denoted by $\Lambda$.

Recall [39] that the Lie algebra of the central extension $\widehat{L G}$ is the product $\widehat{L \mathfrak{g}}=L \mathfrak{g} \times \mathbb{R}$, with bracket

$$
\left[\left(\xi_{1}, t_{1}\right),\left(\xi_{2}, t_{2}\right)\right]=\left(\left[\xi_{1}, \xi_{2}\right], \int_{S^{1}} \operatorname{Tr}\left(\xi_{1} d \xi_{2}\right)\right)
$$

The coadjoint action of $L G$ on $\widehat{L \mathfrak{g}^{*}}:=L \mathfrak{g}^{*} \times \mathbb{R}$ is given by

$$
g \cdot(\xi, \lambda)=\left(\operatorname{Ad}_{g}(\xi)-\lambda d g g^{-1}, \lambda\right)
$$

It follows that the $L G$-actions on elements of $L \mathfrak{g}^{*}$ considered as connections correspond to the action on the affine hyper-plane $L \mathfrak{g}^{*} \times\{1\} \subset \widehat{L \mathfrak{g}^{*}}$. Henceforth we fix a level $\lambda \neq 0$ and identify $L \mathfrak{g}^{*}$ with the affine hyperplane $L \mathfrak{g}^{*} \times\{\lambda\}$.

We choose a closed positive Weyl chamber $\mathfrak{t}_{+} \subset \mathfrak{t}$ and let $\mathfrak{A} \subset \mathfrak{t}_{+}$be the corresponding fundamental alcove. There are natural identifications

$$
\mathfrak{t}_{+} \cong \mathfrak{t} / W \cong \mathfrak{g} / \operatorname{Ad}(G)
$$

that is every (co)adjoint orbit meets the positive Weyl chamber in exactly one point. Similarly, for the affine $L G$-action on $L \mathfrak{g}^{*}$ at level $\lambda$ and the action of $W_{\mathrm{aff}}=W \ltimes \Lambda$ on $\mathrm{t}$ given by $(w, v) \cdot \xi=w \cdot \xi+\lambda v$ one has

$$
\lambda \mathfrak{A} \cong \mathfrak{t} / W_{\text {aff }} \cong L \mathfrak{g}^{*} / L G,
$$

that is, every coadjoint $L G$-orbit at level $\lambda$ meets $\lambda \mathfrak{A} \subset \mathfrak{t} \subset L \mathfrak{g}^{*}$ in exactly one point.

A smooth action of $L G$ on $M$ which preserves the symplectic form will be called Hamiltonian at level $\lambda \in \mathbb{R}$ if there exists a moment map $\Phi: M \rightarrow L \mathfrak{g}^{*}$ such that the composition of $\Phi$ with the inclusion $L \mathfrak{g}^{*} \rightarrow$ 
$\widehat{L \mathfrak{g}^{*}}$ at level $\lambda$ is $L G$-equivariant. Such an action can be considered an action of $\overline{L G}$ with the central circle acting trivially with constant moment map $\lambda$. We emphasize that we require the moment maps for the $L G$-actions to be "sufficiently smooth", i.e. to take values in $L \mathfrak{g}^{*}$, which consists of loops of Sobolev class $s-\frac{1}{2}$. A pre-quantum line bundle for $M$ is $\widehat{L G}$-equivariant with central $S^{1}$ acting with weight $\lambda$. This requires in particular that $\lambda$ is an integer.

The basic examples of Hamiltonian $L G$-spaces (at level $\lambda \neq 0$ ) are coadjoint orbits $\mathcal{O}_{\xi}=L G \cdot(\xi, \lambda)$. Letting $\delta_{\xi / \lambda}$ be the covariant derivative with respect to the connection $\frac{1}{\lambda} \xi$,

$$
\delta_{\xi / \lambda}: L \mathfrak{g} \rightarrow L \mathfrak{g}^{*}, \eta \mapsto d \eta+\frac{1}{\lambda}[\xi, \eta],
$$

the fundamental vector field $\eta_{L \mathfrak{g}^{*}}$ for the infinitesimal action of $L \mathfrak{g}$ on $L \mathfrak{g}^{*}$ at level $\lambda$ is given by $\eta_{L \mathfrak{g}^{*}}(\xi)=-\lambda \delta_{\xi / \lambda}(\eta)$. The symplectic form $\nu_{\xi}$ on $\mathcal{O}_{\xi}$ is given by the usual KKS formula

$$
\nu_{\xi}\left(\left(\eta_{1}\right)_{L \mathfrak{g}^{*}},\left(\eta_{2}\right)_{L \mathfrak{g}^{*}}\right)=\lambda \int_{S^{1}} \operatorname{Tr}\left(\eta_{1} \delta_{\xi / \lambda}\left(\eta_{2}\right)\right)
$$

with moment map as usual the inclusion into $L \mathfrak{g}^{*}$.

We define the (real) weight lattice $\Lambda^{*} \subset \mathfrak{t}^{*}$ to be the dual of the integral lattice. The coadjoint orbit $\mathcal{O}_{\xi}, \xi \in \lambda \mathfrak{A}$ at level $\lambda$ admits a pre-quantum line bundle $\Xi\left(\mathcal{O}_{\xi}\right)$ if and only if $\lambda=m \in \mathbb{Z}$ and $\xi \in \Lambda^{*}$. The set of all such weights

$$
\Lambda_{m}^{*}=m \mathfrak{A} \cap \Lambda^{*}
$$

is called the set of dominant weights at level $m$. For $m \in \mathbb{N}$, geometric quantization of $\Xi\left(\mathcal{O}_{\xi}\right) \rightarrow \mathcal{O}_{\xi}$ by the Borel-Weil construction [39] gives the irreducible positive energy representation of $L G$ at level $m$ with highest weight $\xi$.

\section{Remark 4.1.}

a. Pull-back under the inversion map $I: S^{1} \rightarrow S^{1}, z \mapsto z^{-1}$ induces an automorphism $I^{*}: L G \rightarrow L G$ which transforms a Hamiltonian action with moment map $(\Phi, \lambda)$ into one with moment map $\left(I^{*} \Phi,-\lambda\right)$. We refer to the embedding $\left(1, I^{*}\right): L G \rightarrow L G \times L G$ as the diagonal embedding. If $M$ is a Hamiltonian $L G \times L G$-manifold with moment map $\left(\Phi_{+}, \lambda ; \Phi_{-}, \lambda\right)$ at level $\lambda \neq 0$, then the diagonal action of $L G$ is at level 0 , with moment map $\Phi_{+}-I^{*} \Phi_{-}$. 
b. If $(M, \omega, \Phi)$ is a Hamiltonian $L G$-Banach manifold at non-zero level $\lambda \neq 0$, then $\left(M, \lambda^{-1} \omega, \lambda^{-1} \Phi\right)$ is a Hamiltonian $L G$-space at level +1 . Henceforth, we will always take the level to be +1 unless specified otherwise, and identify $L \mathfrak{g}^{*}$ with the affine hyper-plane $L \mathfrak{g}^{*} \times\{1\} \subset \widehat{L \mathfrak{g}^{*}}$.

Example 4.2. Let $G$ be connected and simply connected. Let $T^{*} \widehat{L G}$ be the cotangent bundle of the central extension of $L G$. Trivialization by left-invariant one-forms gives a diffeomorphism $T^{*} \widehat{L G} \cong$ $\widehat{L G} \times \operatorname{Hom}(\widehat{L \mathfrak{g}}, \mathbb{R})$ where $\operatorname{Hom}(\widehat{L \mathfrak{g}}, \mathbb{R})$ is the topological dual of $L \mathfrak{g}$. The subset $\hat{X}=\widehat{L G} \times \widehat{L \mathfrak{g}^{*}}$ is a Hamiltonian $\widehat{L G} \times \widehat{L G}$-space, with actions given by

$$
L_{\hat{a}}(\hat{g} ; \xi, \lambda)=(\hat{a} \hat{g} ; \xi, \lambda), \quad R_{\hat{a}}(\hat{g} ; \xi, \lambda)=\left(\hat{g} \hat{a}^{-1} ; \operatorname{Ad}_{\hat{a}}(\xi, \lambda)\right)
$$

and moment maps

$$
\hat{\Phi}^{(L)}(\hat{g} ; \xi, \lambda)=\operatorname{Ad}_{\hat{g}}(\xi, \lambda), \quad \hat{\Phi}^{(R)}(\hat{g} ; \xi, \lambda)=-(\xi, \lambda) .
$$

One obtains a Hamiltonian $L G$-manifold $X$ by reducing $\hat{X}$ with respect to central circle $S^{1}$ at moment level 1 . Then $X \cong L G \times L \mathfrak{g}^{*}$, and the induced left and right actions of $L G$

$$
L_{a}(g, \xi)=(a g, \xi), \quad R_{a}(g, \xi)=\left(g a^{-1}, a \cdot \xi\right)
$$

are Hamiltonian, with moment maps

$$
\Phi^{(L)}(g, \xi)=g \cdot \xi, \quad \Phi^{(R)}(g, \xi)=-\xi .
$$

(Here we can use the involution $I^{*}$ to make $\Phi^{(R)}$ into a moment map at level +1 .) It has the property that for every Hamiltonian $L G$-Banach manifold $M$, the reduced space $M \times X / / L G$ by the diagonal action is symplectomorphic to $M$ itself.

The trivial line bundle $L_{\hat{X}}=\hat{X} \times \mathbb{C}$ is a pre-quantum line bundle for $\hat{X}$, with the restriction of the canonical 1-form on $T^{*} \widehat{L G}$ defining a pre-quantum connection. Reduction with respect to the $S^{1}$-action gives an $L(G \times G)$-equivariant pre-quantum bundle $L_{X} \rightarrow X$ isomorphic to the pull-back of $\widehat{L G} \times{ }_{S^{1}} \mathbb{C}$. If $L \rightarrow M$ is an $L G$-equivariant pre-quantum line bundle, the diagonal reduction $L \otimes L_{X} / / L G$ is isomorphic to $L$ itself.

The $L G$-manifold $X$ has a description as a moduli space of flat connections. By the holonomy description, the moduli space $\mathcal{M}\left(\Sigma_{0}^{2}\right)$ of the two-punctured sphere (annulus) is equivariently diffeomorphic to 
the subset $G \times L \mathfrak{g}^{*} \times L \mathfrak{g}^{*} \ni\left(c, \xi_{1}, \xi_{2}\right)$ defined by $\operatorname{Hol}\left(\xi_{1}\right) \operatorname{Ad}_{c} \operatorname{Hol}\left(\xi_{2}\right)=1$. The map

$$
X \rightarrow \mathcal{M}\left(\Sigma_{0}^{2}\right),(g, \eta) \mapsto(g(0), g \cdot \eta,-\eta)
$$

is an equivariant diffeomorphism, preserving the moment maps. Since $\mathcal{M}\left(\Sigma_{0}^{2}\right)$ and $X$ are multiplicity free, i.e., since all reduced spaces $X_{\xi_{1}, \xi_{2}}$ are points, this map is necessarily a symplectomorphism.

\subsection{Symplectic cross-sections}

Hamiltonian loop group actions on Banach manifolds with proper moment maps at positive level behave in many respects like Hamiltonian actions of compact groups on finite dimensional symplectic manifolds. This is due to the existence of finite-dimensional "symplectic crosssections".

\section{Cross-sections for Hamiltonian actions of compact groups}

First we review the symplectic cross-section theorem for actions of compact, connected (not necessarily simply-connected) Lie groups $G$. Choose a maximal torus $T \subset G$ and positive Weyl chamber $\mathfrak{t}_{+}^{*} \subset \mathfrak{t}^{*}$ so that $\mathfrak{g}^{*} / G \cong \mathfrak{t}_{+}^{*}$. For every open face $\sigma$ of $\mathfrak{t}_{+}^{*}$, the stabilizer subgroup $G_{\xi} \subset G$ does not depend on the choice of $\xi \in \sigma$, and is denoted by $G_{\sigma}$. Since $G_{\sigma}$ contains $T$, there is a unique $G_{\sigma}$-invariant splitting of Lie algebras

$$
\mathfrak{g}=\mathfrak{g}_{\sigma} \oplus \mathfrak{g}_{\sigma}^{\perp},
$$

where $\mathfrak{g}_{\sigma}^{\perp}$ is a $G_{\sigma}$-invariant complement. In fact, using an invariant inner product to identify $\mathfrak{g}^{*}$ with $\mathfrak{g}$ and view $\xi$ as an element of $\mathfrak{g}, \mathfrak{g}_{\sigma}$ and $\mathfrak{g}_{\sigma}^{\perp}$ are respectively characterized as the kernel and image of the operator $\operatorname{ad}_{\xi}=[\xi, \cdot]$.

Let $U_{\sigma} \subset \mathfrak{g}^{*}$ be the $G_{\sigma}$-invariant open subset of $\mathfrak{g}_{\sigma}^{*}$ defined by

$$
U_{\sigma}:=G_{\sigma} \cdot \bigcup_{\sigma \subseteq \bar{\tau}} \tau
$$

Then $G \cdot U_{\sigma} \cong G \times_{G_{\sigma}} U_{\sigma}$, which implies that $U_{\sigma}$ is a slice for the coadjoint action at any $x \in \sigma$. Now let $N$ be a Hamiltonian $G_{\sigma}$-space with moment map $\Phi_{N}$. The symplectic induction construction introduced in Guillemin-Sternberg [20] shows that if $\Phi_{N}(N)$ is contained in $U_{\sigma}$, then there exists a unique symplectic structure on the associated bundle

$$
\operatorname{Ind}_{G_{\sigma}}^{G}(N):=G \times_{G_{\sigma}} N
$$


such that the $G$-action is Hamiltonian and the symplectic form $\omega$ and moment map $\Phi$ restrict to the given symplectic form and moment map on $N$. If the $G_{\sigma}$-action on $N$ extends to a Hamiltonian $G_{\sigma} \times K$-action, where $K$ is another compact Lie group, one obtains a Hamiltonian $G \times$ $K$-action on $\operatorname{Ind}_{G_{\sigma}}^{G}(N)$. In particular, the action of $G$ always extends to an action of $G \times Z\left(G_{\sigma}\right)$, where $Z\left(G_{\sigma}\right)$ is the center of $G_{\sigma}$. The moment map for the $Z\left(G_{\sigma}\right)$-action is given by the composition of $\Phi$ with the quotient map $q: \mathfrak{g}^{*} \rightarrow \mathfrak{t}_{+}^{*}$, followed by projection $\mathfrak{t}^{*} \rightarrow \mathfrak{z}\left(\mathfrak{g}_{\sigma}\right)^{*}$.

The symplectic cross-section theorem [20] asserts that conversely, for every Hamiltonian $G$-manifold $M$ with moment map $\Phi: M \rightarrow \mathfrak{g}^{*}$, the pre-image $Y_{\sigma}=\Phi^{-1}\left(U_{\sigma}\right)$ is a $G_{\sigma}$-invariant symplectic submanifold. Consequently, the action of $G$ on $G \cdot Y_{\sigma}$ extends to a Hamiltonian action of $G \times Z\left(G_{\sigma}\right)$. We refer to these $Z\left(G_{\sigma}\right) \subseteq T$-actions (due to GuilleminSternberg [19]) as the induced (toric) actions and to the map

$$
\tilde{\Phi}=q \circ \Phi: M \rightarrow t_{+}^{*}
$$

as the induced (toric) moment map. If $E \rightarrow M$ is a $G$-equivariant vector bundle, the action of $Z\left(G_{\sigma}\right)$ on the restriction $E \mid \Phi^{-1}\left(U_{\sigma}\right)$ extends by $G$-equivariance to an action of $G \times Z\left(G_{\sigma}\right)$ on the restriction of $E$ to $G \cdot \Phi^{-1}\left(U_{\sigma}\right)$.

Suppose now that $M$ is a Hamiltonian $G \times G$-manifold (e.g. a product of Hamiltonian $G$-manifolds) with moment map $\Phi=\left(\Phi_{+}, \Phi_{-}\right)$, and let $M_{0}=M / / G$ be the symplectic quotient by the diagonal action. Since the induced toric moment map $\tilde{\Phi}_{+}$is $G$-invariant, it descends to a map $\tilde{\Phi}_{0}: M_{0} \rightarrow t_{+}^{*}$ which we will call the residual toric moment map. The symplectic cross-section $Y_{\sigma,-\sigma}=\Phi^{-1}\left(U_{\sigma} \times-U_{\sigma}\right)$ is a Hamiltonian $G_{\sigma} \times G_{\sigma}$-space, with moment map the restriction of $\Phi$. There is canonical isomorphism of (possibly singular) symplectic quotients

$$
M_{0} \supset \tilde{\Phi}_{0}^{-1}\left(U_{\sigma} \cap \mathfrak{t}_{+}^{*}\right) \cong Y_{\sigma,-\sigma} / / G_{\sigma} .
$$

\section{Cross-sections for Hamiltonian actions of loop groups}

We now turn to the discussion of symplectic induction and cross-sections for Hamiltonian $L G$-actions on symplectic Banach manifolds, where $G$ is connected and simply connected. We first need to summarize some properties of the coadjoint action of $L G$ on $L \mathfrak{g}^{*}$ at level 1 . Recall [39] that the evaluation map $L G \rightarrow G, g \mapsto g(0)$ maps the isotropy group $(L G)_{\xi}$ of a point $\xi \in L \mathfrak{g}^{*}$ isomorphically to the stabilizer $G_{\mathrm{Hol}(\xi)}$ of 
the holonomy of $\xi$, in particular $(L G)_{\xi}$ is compact and connected ${ }^{2}$. For points $\xi \in \mathfrak{A} \subset \mathfrak{t} \subset L \mathfrak{g}^{*}$, the inverse map is given by $G_{\mathrm{Hol}(\xi)} \mapsto$ $(L G)_{\xi}, k \mapsto \operatorname{Ad}_{\exp (-t \xi)} k$.

It follows that the isotropy group $(L G)_{\xi}$ of a point $\xi \in \mathfrak{A}$ contains $T \subset L G$, and depends only on the open face $\sigma \subset \mathfrak{A}$ containing it. We denote this group by $(L G)_{\sigma}$, the group $G_{\mathrm{Hol}(\xi)}$ by $\overline{(L G)_{\sigma}}$ and the restriction of the central extension $\widehat{L G}$ to $(L G)_{\sigma}$ by ${\widehat{(L G)_{\sigma}}}_{\sigma}$. Note that

$$
\tau \subseteq \bar{\sigma} \Rightarrow(L G)_{\sigma} \subseteq(L G)_{\tau}
$$

If $\bar{\sigma}$ contains 0 , then $(L G)_{\sigma}$ is contained in the subgroup $G \subset L G$ of constant loops and is equal to the stabilizer group of points in $\sigma$ under the coadjoint action of $G$ on $\mathfrak{g}^{*}$.

Another consequence of the above description is that $(L G)_{\sigma}$ consists only of smooth maps. Alternatively, this follows from the fact that its Lie algebra $(L \mathfrak{g})_{\sigma}$ of $(L G)_{\sigma}$ is equal to the kernel of the elliptic operator $\delta_{\xi}: L \mathfrak{g} \rightarrow L \mathfrak{g}^{*}$ defined in (16). The image $\operatorname{im}\left(\delta_{\xi}\right)$ is equal to the tangent space to the $L G$-orbit through $\xi$, i.e., to the annihilator $(L \mathfrak{g})_{\sigma}^{0}$, so that there are $(L G)_{\sigma}$-invariant direct sum (Hodge) decompositions

$$
L \mathfrak{g}^{*}=(L \mathfrak{g})_{\sigma}^{*} \oplus(L \mathfrak{g})_{\sigma}^{0}
$$

and dually

$$
L \mathfrak{g}=(L \mathfrak{g})_{\sigma} \oplus(L \mathfrak{g})_{\sigma}^{\perp} .
$$

The map $\delta_{\xi}$ induces a $(L G)_{\sigma}$-equivariant Banach space isomorphism $(L \mathfrak{g})_{\sigma}^{\perp} \cong(L \mathfrak{g})_{\sigma}^{0}$.

Remark 4.3. It is important to note that since the $L G$-action on $L \mathfrak{g}^{*}$ is only affine-linear, the action of $(L G)_{\sigma}$ on

$$
(L \mathfrak{g})_{\sigma}^{*} \cong(L \mathfrak{g})_{\sigma}^{*} \times\{1\} \subset \widehat{L \mathfrak{g}^{*}}
$$

in this splitting is not the coadjoint action unless $0 \in \bar{\sigma}$. Indeed, from the description of the isomorphism $(L G)_{\sigma} \cong G_{\mathrm{Hol}(\xi)}$ and the coadjoint action of $\widehat{L G}$ given in (15) one finds that the action is given by

$$
(L G)_{\sigma} \times(L \mathfrak{g})_{\sigma}^{*} \rightarrow(L \mathfrak{g})_{\sigma}^{*}, \quad(k, \eta) \mapsto\left(\operatorname{Ad}_{k^{-1}}\right)^{*}(\eta-\mu)+\mu
$$

where $\mu$ is any element in the affine span of $\sigma$.

\footnotetext{
${ }^{2}$ The stabilizer groups for the conjugation action of a compact, connected, simply connected Lie group on itself are connected by a result of Bott-Samelson described in [11].
} 
As above, we now define, for every open face $\sigma \subset \mathfrak{A}$

$$
U_{\sigma}=(L G)_{\sigma} \cdot \bigcup_{\sigma \subseteq \bar{\tau}} \tau
$$

Note that $U_{\sigma}$ is an open subset of $(L \mathfrak{g})_{\sigma}^{*}$, in particular it is finite dimensional and consists only of smooth elements of $L \mathfrak{g}^{*}$.

Lemma 4.4. The set $U_{\sigma}$ is a slice for all $\xi \in \sigma$ for the action of $L G$, i.e., the canonical map

$$
L G \times{ }_{(L G)_{\sigma}} U_{\sigma} \rightarrow L G \cdot U_{\sigma}
$$

is a diffeomorphism of Banach manifolds.

Proof. The map is bijective because for any $\eta \in U_{\sigma}$, the stabilizer $(L G)_{\eta} \subseteq(L G)_{\sigma}$. That the differential is an isomorphism follows from the splitting (19). q.e.d.

Remark 4.5. Let $\sigma=\{\xi\} \subset \mathfrak{A}$ be a vertex such that $\exp (-\xi)$ is contained in the center $Z(G)$ of $G$, i.e., $\overline{(L G)_{\sigma}}=G$. (For example, if $G=S U(n)$ all vertices of $\mathfrak{A}$ exponentiate to elements of the center.) Then $f_{\xi}(t):=\exp (-t \xi)$ defines an exterior automorphism of $L G$,

$$
\left(f_{\xi} \cdot g\right)(t)=\operatorname{Ad}_{f_{\xi}(t)} g(t)
$$

Similarly, there is an automorphism of $L \mathfrak{g}^{*}$ by

$$
f_{\xi} \cdot \eta=\operatorname{Ad}_{f_{\xi}(t)}(\eta)+\xi \mathrm{d} t
$$

These two automorphisms are compatible, that is

$$
f_{\xi} \cdot(g \cdot \eta)=\left(f_{\xi} \cdot g\right) \cdot\left(f_{\xi} \cdot \eta\right) .
$$

It follows that $f_{\xi}$ induces an equivariant diffeomorphism the slice $U_{\{0\}} \rightarrow$ $U_{\sigma}$.

Lemma 4.6. For all $\xi \in U_{\sigma}$, the tangent space $T_{\xi}(L G \cdot \xi) \cong \operatorname{im}\left(\delta_{\xi}\right)$ to the coadjoint orbit $\mathcal{O}_{\xi}$ through $\xi$ decomposes into an $\nu_{\xi}$-orthogonal direct sum of closed symplectic subspaces,

$$
T_{\xi}(L G \cdot \xi) \cong T_{\xi}\left((L G)_{\sigma} \cdot \xi\right) \oplus(L \mathfrak{g})_{\sigma}^{0}
$$

where $(L \mathfrak{g})_{\sigma}^{0}$ is the annihilator of $(L \mathfrak{g})_{\sigma}$ in $L \mathfrak{g}^{*}$. 
Proof. It follows easily from the definition (17) of the symplectic form on $L G \cdot \xi$ that the two subspaces are symplectically orthogonal. The proof is completed by noting that the coadjoint orbit $(L G)_{\sigma} \cdot \xi$ with the KKS form is a symplectic submanifold. q.e.d.

Theorem 4.7 (Symplectic induction). Let $\sigma$ be a face of $\mathfrak{A}$ and $N$ a symplectic Banach manifold with a Hamiltonian $(L G)_{\sigma}$-action and moment map $\Phi_{N}: N \rightarrow(L \mathfrak{g})_{\sigma}^{*}$ such that for some $\mu$ in the affine span of $\sigma$, the image $\left(\Phi_{N}+\mu\right)(N) \subset U_{\sigma}$. Then there exists a unique $L G$ invariant symplectic form $\omega$ on the Banach manifold

$$
\operatorname{Ind}_{(L G)_{\sigma}}^{L G}(N):=L G \times{ }_{(L G)_{\sigma}} N
$$

such the LG-action is Hamiltonian, with moment map

$$
\Phi: \operatorname{Ind}_{(L G)_{\sigma}}^{L G}(N) \rightarrow L \mathfrak{g}^{*}
$$

and such that the pull-back of $\omega$ (resp. $\Phi$ ) to $N$ is equal to the given symplectic form $\omega_{N}$ (resp. moment map $\Phi_{N}+\mu$ ) on $N$.

Proof. Let $M:=L G \times(L G)_{\sigma} N$. Since $(L G)_{\sigma}$ is compact and acts freely on $L G, M$ is a Banach manifold. By Equation (21) the map $\Phi_{N}+\mu: N \rightarrow(L \mathfrak{g})_{\sigma}^{*} \oplus\{1\}$ extends to a unique $L G$-equivariant map $\Phi: M \rightarrow L G \times \times_{(L G)_{\sigma}}(L \mathfrak{g})_{\sigma}^{*} \hookrightarrow L \mathfrak{g}^{*}$. The moment map condition

$$
\iota\left(\eta_{M}\right) \omega_{x}=d\langle\Phi, \eta\rangle
$$

$L G$-invariance of $\omega$ and the condition $\iota_{N}^{*} \omega=\omega_{N}$ completely determine $\omega$, and also imply that $\omega$ is closed. To show $M$ is symplectic let $x \in N \subset M$, and $\tau \subset \mathfrak{A}$ the open face containing $\Phi_{N}(x)$. By (22) together with Lemma 4.6, there is a natural $\omega$-orthogonal splitting $T_{x} M \cong T_{x} N \oplus(L \mathfrak{g})_{\sigma}^{0}$. Since $\sigma \subseteq \bar{\tau}$, the form $\omega_{x}$ is non-degenerate on the second summand, which implies that $T_{x} M$ is symplectic. q.e.d.

Theorem 4.8 (Symplectic cross-section). Let $(M, \omega)$ be a symplectic Banach manifold, and $L G \times M \rightarrow M$ a Hamiltonian $L G$-action with moment map $\Phi: M \rightarrow L \mathfrak{g}^{*}$. For every open face $\sigma \subset \mathfrak{A}$, the symplectic cross-section $Y_{\sigma}:=\Phi^{-1}\left(U_{\sigma}\right)$ is a symplectic $(L G)_{\sigma}$-invariant Banach submanifold, and the action of $(L G)_{\sigma}$ is Hamiltonian. The restriction $\Phi \mid Y_{\sigma}$ is a moment map for the action of $\widehat{(L G)_{\sigma}}$, and a moment map for the $(L G)_{\sigma}$-action is given by $\Phi \mid Y_{\sigma}-\mu$, for any $\mu$ in the affine span of $\sigma$. If $\Phi$ is proper, $Y_{\sigma}$ is finite-dimensional. 
Proof. By equivariance and since $U_{\sigma}$ is a slice for the $L G$-action, $\Phi$ is transversal to $U_{\sigma}$. The implicit function theorem for Banach manifolds thus shows that $N:=\Phi^{-1}\left(U_{\sigma}\right)$ is a smooth Banach submanifold. Since $T_{\Phi(x)} U_{\sigma}=(L \mathfrak{g})_{\sigma}^{*} \subset L \mathfrak{g}^{*}$, the tangent space $T_{x} N$ at some $x \in N$ is equal to $\left(\mathrm{d}_{x} \Phi\right)^{-1}\left((L \mathfrak{g})_{\sigma}^{*}\right)$. In order to show that $T_{x} N$ is symplectic, we show that $T_{x} M \cong E \oplus T_{x} N$ where $E$ is a closed symplectic complement to $T_{x} N$ that is symplectically perpendicular to $T_{x} N$.

Let $E$ be the image of the map $(L \mathfrak{g})_{\sigma}^{\perp} \rightarrow T_{x} M$ sending $\eta$ to the fundamental vector field $\eta_{M}(x)$. A continuous inverse is given by the composition of the $\operatorname{map}_{x} \Phi: E \rightarrow(L \mathfrak{g})_{\sigma}^{0}$ with the isomorphism $(L \mathfrak{g})_{\sigma}^{0} \cong(L \mathfrak{g})_{\sigma}^{\perp}$. Hence $E$ is a closed complement to $T_{x} N$.

By equivariance of the moment map, one has for all $\eta, \zeta \in L \mathfrak{g}$,

$$
\omega_{x}\left(\eta_{M}, \zeta_{M}\right)=\nu_{\xi}\left(\eta_{L \mathfrak{g}^{*}}, \zeta_{L \mathfrak{g}^{*}}\right)=\int_{S^{1}} \operatorname{Tr}\left(\eta \delta_{\xi} \zeta\right)
$$

By Lemma $4.6,(L \mathfrak{g})_{\sigma}^{0}$ is a symplectic subspace of $T_{\Phi(x)}(L G \cdot \Phi(x))$. It follows that $\mathrm{d}_{x} \Phi$ restricts to a symplectic isomorphism from $E$ to $(L \mathfrak{g})_{\sigma}^{0}$, and also that $T_{x} N$ is symplectically perpendicular to $E$.

The restriction of $\Phi$ to $Y_{\sigma}$ is a moment map for the $(L G)_{\sigma}$-action which is equivariant with respect to the affine action on $(L \mathfrak{g})_{\sigma}^{*} \oplus\{1\}$ given in Equation (21). Hence, subtracting $\mu$ gives a moment map which is equivariant with respect to the usual coadjoint action. q.e.d.

In particular this shows that Hamiltonian actions of loop groups at non-zero level are always proper group actions.

\section{Cross-section for moduli spaces}

In the case of moduli spaces of flat connections, the cross-sections $Y_{\sigma}$ are open subsets of the extended moduli spaces of Jeffrey [23] and Huebschmann [21]. Let $\Sigma$ be a compact, connected, oriented 2-manifold of genus $g$ with $b$ boundary components. Consider once again the Hamiltonian $L G^{b}$-manifold $\mathcal{M}(\Sigma)$ for $b>0$. In the holonomy description, the cross-sections $Y_{\sigma_{1} \ldots \sigma_{b}}$ for faces $\sigma=\left(\sigma_{1}, \ldots, \sigma_{b}\right)$ of $\mathfrak{A}^{b}$ are the smooth submanifolds of $G^{2 g} \times G^{b-1} \times\left(L \mathfrak{g}^{*}\right)^{b}$ given by the condition (8) together with the requirement $\xi_{j} \in U_{\sigma_{j}}$. For the dimension of $Y_{\sigma_{1} \ldots \sigma_{j}}$, we find

$$
\begin{aligned}
\operatorname{dim}\left(Y_{\sigma_{1} \ldots \sigma_{j}}\right) & =(2 g+b-1) \operatorname{dim} G+\sum \operatorname{dim} U_{\sigma_{j}}-\operatorname{dim} G \\
& =(2 g-2) \operatorname{dim} G+\sum\left(\operatorname{dim} G+\operatorname{dim}(L G)_{\sigma_{j}}\right) .
\end{aligned}
$$


The extended moduli spaces of Jeffrey [23] and Huebschmann [21] are defined, for a 2-manifold with a single boundary component, as the subset of $G^{2 g} \times \mathfrak{g}^{*}$ given by the condition $\prod\left[a_{2 i-1}, a_{2 i}\right]=\exp (\xi)$. On a neighborhood of $\xi=0$, this is a smooth submanifold and can be given a symplectic structure; however for larger $\xi$ one encounters singularities and degeneracies of the symplectic form. One can view the extended moduli space as the symplectic cross-section corresponding to $\sigma=\{0\}$. The singularities of the extended moduli spaces find a natural explanation in the full space $\mathfrak{g} \subset L \mathfrak{g}^{*}$ not being a slice for $L G$. In Jeffrey [23], more general extended moduli spaces are defined for all central elements $c \in Z(G)$ by the modified condition $\prod\left[a_{2 i-1}, a_{2 i}\right]=c \exp (\xi)$; these may be identified, using Remark 4.5, with the cross-sections corresponding to $\sigma=\{\eta\}$, where $\eta \in \mathfrak{A}$ is the unique vertex such that $\exp (-\eta)=c$. Given a Riemannian metric on $\Sigma$ the moduli space $\mathcal{M}(\Sigma)$ becomes a Banach-Kähler manifold; however the symplectic cross-sections are not Kähler submanifolds.

\subsection{Symplectic reduction of Hamiltonian $L G$-manifolds}

The existence of finite-dimensional symplectic cross-sections allows us to prove the main result of this section, Proposition 4.9 below, which states that a diagonal reduction of a Hamiltonian $L G \times L G$-manifold with proper moment map may be written locally as a finite-dimensional symplectic reduction. Let $M$ be a Hamiltonian $L(G \times G)$-manifold with moment map $\left(\Phi_{+}, \Phi_{-}\right)$. We define cross-sections

$$
\left.Y_{\sigma,-\sigma}:=\left(\Phi_{+}, \Phi_{-}\right)^{-1}\left(U_{\sigma} \times I^{*} U_{\sigma}\right)\right) .
$$

which are Hamiltonian $(L G)_{\sigma} \times I^{*}(L G)_{\sigma}$-spaces. Note that the diagonal action of $(L G)_{\sigma} \subset L G$ on $Y_{\sigma,-\sigma}$ is Hamiltonian with moment map the restriction of $\Phi_{+}-I^{*} \Phi_{-}$, i.e., no shift is required.

As in the finite dimensional case, every Hamiltonian $L G$-manifold has Hamiltonian actions (induced toric flows) of the centers $Z\left((L G)_{\sigma}\right)$ on $L G \cdot Y_{\sigma}$ that commute with the action of $L G$. The moment maps for these actions are given as the composition of the induced toric moment map

$$
\tilde{\Phi}:=q \circ \Phi: M \rightarrow \mathfrak{A},
$$

with the projection $\mathfrak{t}^{*} \rightarrow \mathfrak{z}\left((L \mathfrak{g})_{\sigma}\right)^{*}$. Here $q: L \mathfrak{g}^{*} \rightarrow \mathfrak{A}=L \mathfrak{g}^{*} / L G$ is the quotient map, which can also be written as the composition of the holonomy map with the quotient $\operatorname{map} G \rightarrow G / \operatorname{Ad}(G)$. As before, if 
$M=\hat{M} / / L G$ is the symplectic reduction of a Hamiltonian $L(G \times G)$ manifold $\hat{M}$ with moment map $\left(\Phi_{+}, \Phi_{-}\right)$by the diagonal action, the maps $\tilde{\Phi}_{ \pm}$for $\hat{M}$ descend to a map $\tilde{\Phi}: M \rightarrow \mathfrak{A}$, whose $\mathfrak{z}\left((L \mathfrak{g})_{\sigma}\right)^{*}$ component is a moment map for the induced action of $Z\left((L G)_{\sigma}\right)$ on $\tilde{\Phi}^{-1}\left(U_{\sigma} \cap \mathfrak{A}\right)$. We refer to these as the residual toric moment map and residual toric flow.

Proposition 4.9. Let $H, G$ be compact connected simply connected groups and $\mathfrak{A}$ the fundamental alcove for $G$. Let $M$ be a Hamiltonian $L(H \times G \times G)$-Banach manifold, $M_{0}:=M / / L G$ the (possibly singular) reduction by the diagonal $L G$-action, and $\tilde{\Phi}_{0}: M_{0} \rightarrow \mathfrak{A}$ the residual toric moment map. For every face $\sigma$ of $\mathfrak{A}$ we have a canonical homeomorphism

$$
\tilde{\Phi}_{0}^{-1}\left(U_{\sigma}\right) \cong Y_{\sigma,-\sigma} / /(L G)_{\sigma} .
$$

If the diagonal action of $L G$-action is free on the zero level set, then $M_{0}$ is a smooth Hamiltonian LH-Banach manifold and the above identification is a symplectomorphism. If $H=\{1\}$ and if the moment map is proper then $M_{0}$ is finite dimensional.

Proof. Note that $\tilde{\Phi}_{0}^{-1}\left(U_{\sigma}\right)$ is equal to the open subset

$$
\left(L G^{2} \cdot Y_{\sigma,-\sigma}\right) / / L G \subseteq M_{0}
$$

Since $L G^{2} \cdot Y_{\sigma,-\sigma}$ is symplectomorphic to the symplectic induction

$$
\operatorname{Ind}_{\left(L G^{2}\right)_{\sigma, \sigma}}^{L G^{2}}\left(Y_{\sigma,-\sigma}\right)=L G^{2} \times_{\left(L G^{2}\right)_{\sigma, \sigma}} Y_{\sigma,-\sigma}
$$

the formula (23) follows. Now suppose that the diagonal $L G$-action on the zero level set is free. Then the diagonal action of $(L G)_{\sigma}$ on the zero level set in $Y_{\sigma,-\sigma}$ is free. Since $(L G)_{\sigma}$ is compact, this implies that 0 is a regular value for the moment map for both of these actions, and (23) is a diffeomorphism of Banach manifolds. Also, it is clear that the 2 -forms induced by the symplectic form $\omega$ on both sides of (23) coincide. Since the Marsden-Weinstein theorem holds for Hamiltonian actions of compact groups on Banach manifolds, it follows that the 2-form on $M_{0}$ is symplectic. q.e.d.

Let $G$ be a compact connected simply connected Lie group, with fundamental alcove $\mathfrak{A}$, and let $M$ be a Hamiltonian $L G$-Banach manifold. For each $\xi \in \mathfrak{A}$ let $\mathcal{O}_{\xi}=L G \cdot \xi$ be the corresponding loop group 
orbit. Define the reduction of $M$ at $\xi$ as the symplectic reduction by the diagonal $L G$-action

$$
M_{\xi}=M \times \mathcal{O}_{* \xi} / / L G
$$

Letting $\sigma$ be a face of $\mathfrak{A}$ such that $\xi \in U_{\sigma}$ and $Y_{\sigma}$ the corresponding symplectic cross-section of $M$, one also has

$$
M_{\xi}=Y_{\sigma} \times \mathcal{O}_{* \xi}^{\prime} / /(L G)_{\sigma}
$$

where $\mathcal{O}_{* \xi}^{\prime}=\mathcal{O}_{* \xi} \cap\left((L \mathfrak{g})_{\sigma}^{*} \times\{1\}\right)=\widehat{(L G)_{\sigma}} \cdot * \xi$ is the orbit for the compact group $\widehat{(L G)_{\sigma}}$. From (24), one finds in particular that if the moment map is proper, then $M_{\xi}$ is a finite dimensional symplectic quotient. In the case that the quotient is singular, the local structure of its singularities can be described by normal form theorems as in Sjamaar-Lerman [43].

If $M$ is a Hamiltonian $L G$-manifold at integer level $m>0, L \rightarrow M$ an $\widehat{L G}$-equivariant pre-quantum line bundle, and $\xi \in \Lambda_{m}^{*}$, then we define

$$
L_{\xi}:=L \otimes \Xi\left(\mathcal{O}_{* \xi}\right) / / L G
$$

where $\Xi\left(\mathcal{O}_{* \xi}\right)$ is the pre-quantum line bundle of $\mathcal{O}_{* \xi}$ as in Section 4.1. If the diagonal action of $L G$ is locally free on the zero level set, then $L_{\xi}$ is a pre-quantum (orbifold) line bundle over $M_{\xi}$.

The quotients $\mathcal{M}(\Sigma)_{\xi_{1}, \ldots, \xi_{b}}$ of $\mathcal{M}(\Sigma)$ are the moduli spaces of flat connections with fixed holonomies around the boundary components. By the above discussion, these are compact symplectic stratified spaces. There is a holonomy description for these moduli spaces, as in the case without markings:

Theorem 4.10. Let $\xi_{1}, \ldots, \xi_{b} \in \mathfrak{A}$, and $\mathcal{C}_{1}, \ldots, \mathcal{C}_{b} \subset G$ the corresponding conjugacy classes. The moduli space $\mathcal{M}(\Sigma)_{\xi_{1}, \ldots, \xi_{b}}$ is homeomorphic to the quotient of the subset $(a, d) \in G^{2 g} \times \mathcal{C}_{1} \times \ldots \times \mathcal{C}_{b}$ given by

$$
\prod_{i=1}^{g}\left[a_{2 i-1}, a_{2 i}\right]=\prod_{j=1}^{b} d_{j}
$$

by the conjugation action of $G$. 


\subsection{Convexity theorems for Hamiltonian loop group ac- tions}

In the following theorem, we use symplectic cross-sections to derive convexity and connectedness properties for the moment map of Hamiltonian $L G$-manifolds. Similar results were announced by S. Chang several years ago.

Theorem 4.11. Let $G$ be a compact connected simply connected Lie group, and $M$ a connected Hamiltonian LG-manifold with proper moment map $\Phi: M \rightarrow L \mathfrak{g}^{*}$.

a. For any face $\sigma$ of the fundamental alcove $\mathfrak{A}$ such that $\Phi(M) \cap \sigma \neq$ $\emptyset$, the corresponding symplectic cross-section $Y_{\sigma}$ is finite dimensional and connected.

$b$. The fibers of $\Phi$ are connected.

c. The intersection $\Phi(M) \cap \mathfrak{A}$ is a convex polytope.

Proof. Finite dimensionality follows from the fact that the restriction of $\Phi$ to the submanifold $Y_{\sigma}=\Phi^{-1}\left(U_{\sigma}\right)$ is proper as a map to $U_{\sigma}$. Since $U_{\sigma}$ is finite dimensional, this is only possible if $Y_{\sigma}$ is finite dimensional.

We next prove that all non-empty cross-sections $Y_{\sigma}$ are connected. Since the coadjoint orbits $L G /(L G)_{\sigma}$ are simply connected, this is the case if and only if all flow-outs $L G \cdot Y_{\sigma}=L G \times(L G)_{\sigma} Y_{\sigma}$ are connected. By properness of $\Phi$, the number of connected components $Y_{\sigma}^{i}$ of $Y_{\sigma}$ is finite. The fact that $L G \cdot Y_{\sigma}$ is connected follows once we can show the transitivity property

$$
\begin{gathered}
L G \cdot\left(Y_{\sigma}^{i} \cap Y_{\tau}^{j}\right) \neq \emptyset, \quad L G \cdot\left(Y_{\tau}^{j} \cap Y_{\kappa}^{k}\right) \neq \emptyset \\
\Longrightarrow L G \cdot\left(Y_{\sigma}^{i} \cap Y_{\kappa}^{k}\right) \neq \emptyset,
\end{gathered}
$$

since the collection of all $L G \cdot Y_{\sigma}^{i}$ is a finite open covering of $M$, and any two points in $M$ can be joined by a path.

To show (27), let $x \in Y_{\sigma}^{i} \cap Y_{\tau}^{j}$ and $y \in Y_{\tau}^{j} \cap Y_{\kappa}^{k}$. The fact that the restriction of $\tilde{\Phi}$ to $Y_{\tau}^{j}$ is proper as a map into $U_{\tau}$ implies that it has connected fibers and that the image $\tilde{\Phi}\left(Y_{\tau}^{j}\right) \subset \mathfrak{A}$ is convex (see [33] or the explanation of the Condevaux-Delzord-Molino in Flaschka-Ratiu $[15$, p. 29]). In particular, the line segment from $\alpha=\tilde{\Phi}(x)$ to $\beta=\tilde{\Phi}(y)$. is contained in $\tilde{\Phi}\left(Y_{\tau}^{j}\right)$. It follows that there exists a path $\gamma$ in $L G \cdot Y_{\tau}^{j}$ connecting $x$ and $y$ whose image under $\tilde{\Phi}$ is the line segment $\overline{\alpha \beta}$. The 
interior of $\overline{\alpha \beta}$ intersects $U_{\sigma} \cap \mathfrak{A}$ and $U_{\kappa} \cap \mathfrak{A}$, and is therefore contained in $U_{\sigma} \cap U_{\kappa} \cap \mathfrak{A}$. It follows that $\gamma^{-1}(\operatorname{int}(\overline{\alpha \beta})) \subset L G \cdot\left(Y_{\sigma}^{i} \cap Y_{\kappa}^{k}\right)$.

Since all cross-sections $Y_{\sigma}$ are connected and all restrictions $\Phi \mid Y_{\sigma}$ have connected fibers [33], it follows that $\Phi$ has connected fibers. Since $\Phi$ is proper, the number of $(L G)_{\sigma}$-conjugacy classes of stabilizer groups (orbit types) for the $(L G)_{\sigma}$-action on $Y_{\sigma}$ is finite. By [33, Remark 5.2] or the Condevaux-Delzord-Molino technique, this implies that $\tilde{\Phi}\left(Y_{\sigma}\right)$ is the intersection of $U_{\sigma} \cap \mathfrak{A}$ with a convex polyhedron. Since $M$ is connected, and since a closed connected set is convex if and only if it is locally convex, this shows that the image $\tilde{\Phi}(M)$ is a convex polytope. q.e.d.

Remark 4.12. As far as we know the Atiyah-Pressley convexity Theorem [5], which is a Kostant-type convexity theorem on projections of coadjoint loop group orbits, does not fit into this framework. Note that the relevant moment map, given by projection of the orbit to $t^{*}$ together with the energy function, is not proper.

In the case $M=\mathcal{M}(\Sigma)$, since $[G, G]=G$ the holonomy description also shows that for $g \geq 1$ the moment map is surjective onto $\left(L \mathfrak{g}^{*}\right)^{b}$ so that the moment polytope is simply $\mathfrak{A}^{b}$ and the convexity Theorem 4.11 is vacuous. On the other hand, in the case $g=0$ of a $b$-punctured sphere, the holonomy description of $\mathcal{M}(\Sigma)$ and Theorem 4.11 yield the following.

Corollary 4.13. The image of the subset

$$
\left\{d \in G^{b} \mid \prod_{j=1}^{b} d_{j}=1\right\} \subset G^{b}
$$

under the quotient map $G^{b} \rightarrow \mathfrak{A}^{b}$ is a convex polytope.

For the case $G=S U(n)$, this polytope is described in AgnihotriWoodward [2].

Corollary 4.14. (Convexity properties of the induced toric moment map.) Let $H, G$ be compact connected simply connected Lie groups, with fundamental alcoves $\mathfrak{B}, \mathfrak{A}$, and $M$ be a Hamiltonian $L(H \times G \times G)$ manifold with proper moment map. Let $M_{0}$ be the symplectic reduction with respect to the diagonal LG-action, and $\tilde{\Phi}_{0}: M_{0} \rightarrow \mathfrak{B} \times \mathfrak{A}$ the product of the induced toric moment map for the induced $L H$-action and the residual toric moment map obtained from the $\{e\} \times\{e\} \times L G-$ action on $M$. Then the image of $\tilde{\Phi}_{0}$ is a convex polytope. 
Proof. Let $\Phi: M \rightarrow L(\mathfrak{h} \oplus \mathfrak{g} \oplus \mathfrak{g})^{*}$ be the moment map for the $L(H \times G \times G)$-action, and $\tilde{\Phi}: M \rightarrow \mathfrak{B} \times \mathfrak{A} \times \mathfrak{A}$ the corresponding toric moment map. Consider the involution $\kappa$ of $\mathfrak{B} \times \mathfrak{A} \times \mathfrak{A}$ defined by

$$
\kappa\left(x_{1}, x_{2}, x_{3}\right)=\left(x_{1}, * x_{3}, * x_{2}\right),
$$

where $*$ is the Cartan involution given by $* \mu=q(-\mu)$. The image of the induced map $\tilde{\Phi}_{0}$ is given by the image of the intersection $\tilde{\Phi}(M) \cap$ $(\mathfrak{B} \times \mathfrak{A} \times \mathfrak{A})^{\kappa}$, where $(\mathfrak{B} \times \mathfrak{A} \times \mathfrak{A})^{\kappa}$ denotes the fixed point set of the involution $\kappa$, under the projection of $\mathfrak{B} \times \mathfrak{A} \times \mathfrak{A}$ to the first and second factors. q.e.d.

For every compact connected 2-manifold $\Sigma$ of genus $g$ with $b$ boundary components, except for the cases $(g, b)=(0,0),(0,1),(0,2),(1,0)$, there exist embedded circles $C_{1}, \ldots, C_{r} \subset \Sigma$ that decompose $\Sigma$ into a union of 3-holed spheres (pairs of pants) $\Sigma_{1}, \ldots, \Sigma_{l}$. For the number $l$ of pants and $r$ of separating curves in this decomposition, one has $3 l=b+3 r$, where:

$$
\begin{aligned}
r=3 g+b-6 & \text { if } \quad b \geq 1, g \geq 2 \\
r=3 g-3 & \text { if } \quad b=0, g \geq 2 \\
r=b & \text { if } \quad b \geq 1, g=1 \\
r=b-1 & \text { if } \quad b \geq 3, g=0
\end{aligned}
$$

By repeated application of Theorem 3.5, the moduli space $\mathcal{M}(\Sigma)$ is obtained as a symplectic reduction

$$
\mathcal{M}(\Sigma)=\mathcal{M}\left(\Sigma_{1}\right) \times \ldots \times \mathcal{M}\left(\Sigma_{l}\right) / / L G^{r} .
$$

As in the finite dimensional case, this induces a residual toric flow on $\mathcal{M}(\Sigma)$, i.e., a densely defined, effective Hamiltonian $T^{r+b}$-action which commutes with the action of $\mathcal{G}(\partial \Sigma)$, known as the Goldman twist flow. The corresponding residual toric moment map, called here the Goldman map, is

$\mathfrak{G}: \mathcal{M}(\Sigma) \rightarrow \mathfrak{A}^{r+b}$,

$[A] \rightarrow\left(G \cdot \operatorname{Hol}_{B_{1}}(A), \ldots, G \cdot \operatorname{Hol}_{B_{b}}(A), G \cdot \operatorname{Hol}_{C_{1}}(A), \ldots, G \cdot \operatorname{Hol}_{C_{r}}(A)\right)$.

As a special case of Corollary 4.14, we have:

Corollary 4.15. The image $\mathfrak{G}(\mathcal{M}(\Sigma)) \subset \mathfrak{A}^{b+r}$ of the Goldman map $\mathfrak{G}$ is $a(b+r) \cdot \operatorname{dim} T$-dimensional convex polytope. 
This polytope is given explicitly as follows. Let $P \subset \mathfrak{A}^{3}$ denote the polytope which is the moment polytope for the three-holed sphere. For each $\Sigma_{j}$ let $B_{j}^{1}, B_{j}^{2}, B_{j}^{3} \subset \partial \Sigma_{j}$ denote its boundary components, and let $\mathfrak{A}_{j}^{\nu}$ be a copy of the fundamental alcove associated to $B_{j}^{\nu}$. Define an involution $\kappa: \mathfrak{A}^{3 l} \rightarrow \mathfrak{A}^{3 l}$ by

$$
(\kappa(x))_{j}^{\nu}=\left\{\begin{array}{cc}
x_{l}^{\mu} & \text { if } B_{j}^{\nu}=B_{l}^{\mu} \text { for some }(l, \mu) \\
x_{j}^{\nu} & \text { otherwise. }
\end{array}\right.
$$

Then $\mathfrak{G}(\mathcal{M}(\Sigma))$ is the image of the intersection $P^{l} \cap\left(\mathfrak{A}^{3 l}\right)^{\kappa}$ where $\left(\mathfrak{A}^{3 l}\right)^{\kappa}$ denotes the fixed point set of the involution $\kappa$, under the projection $\mathfrak{A}^{3 l} \rightarrow \mathfrak{A}^{b+r}$.

Remark 4.16. For the case $G=S U(2)$ and $\partial \Sigma=\emptyset$ the dimension $(2 g-2) \operatorname{dim} G$ of the moduli space $\mathcal{M}(\Sigma)$ is precisely twice the dimension of this polytope, which means that the Goldman flow gives a completely integrable torus action on a dense subset of $\mathcal{M}(\Sigma)$. This has been studied extensively in Jeffrey-Weitsman [22].

\section{5. $\operatorname{Spin}_{c}$ quantization}

In this section, we define the $\operatorname{Spin}_{c}$ quantization, and introduce techniques for dealing with singular quotients.

\subsection{Riemann-Roch numbers for almost complex orbifolds}

Let $M$ be a compact almost complex $G$-manifold. Then $M$ has a canonical $G$-invariant $\operatorname{Spin}_{c}$-structure. Given a Hermitian line bundle $L \rightarrow M$, one can consider the G-equivariant $\operatorname{Spin}_{c}$-Dirac operator $\not_{L}$ corresponding to this $\mathrm{Spin}_{c}$-structure, twisted by $L$ (see e.g. [31]). The equivariant index of $\not_{L}$ is a virtual character of $G$ which we call the equivariant Riemann-Roch number,

$$
\operatorname{RR}(M, L):=\operatorname{ind}_{G}\left(\not \partial{ }_{L}\right) \in \operatorname{Rep}(G),
$$

where $\operatorname{Rep}(G)$ denotes the representation ring for $G$. An explicit expression for $\operatorname{RR}(M, L)$ is given by the Equivariant Index Theorem of Atiyah-Segal-Singer.

If $M$ is an almost complex $G$-orbifold, and $L \rightarrow M$ a $G$-orbifold line bundle the same definition applies. A formula for the character is given by the orbifold index theorem of Kawasaki [25] (the equivariant version 
of which was proved by Vergne [50]). Riemann-Roch numbers satisfy the following functorial properties:

a. (Products) If $L_{i} \rightarrow M_{i}$ are $G_{i}$-equivariant line bundles over compact almost complex $G_{i}$-orbifolds $(i=1,2)$, then

$$
\mathrm{RR}\left(M_{1} \times M_{2}, L_{1} \otimes L_{2}\right)=\mathrm{RR}\left(M_{1}, L_{1}\right) \otimes \mathrm{RR}\left(M_{2}, L_{2}\right) .
$$

b. (Conjugation) Let $L \rightarrow M$ be a $G$-equivariant orbifold bundle over a compact almost complex $G$-orbifold. Let $M^{*}$ denote $M$ with the opposite almost complex structure. Then

$$
\mathrm{RR}\left(M^{*}, L^{*}\right)=\mathrm{RR}(M, L)^{*},
$$

where, for all $\chi \in \operatorname{Rep}(G), \chi^{*} \in \operatorname{Rep}(G)$ denotes the dual representation.

c. (Induction) Let $\sigma \subset \mathfrak{t}_{+}^{*}$ be an open face of the positive Weyl chamber, with corresponding stabilizer group $G_{\sigma}$. Let

$$
\operatorname{Ind}_{G_{\sigma}}^{G}: \operatorname{Rep}\left(G_{\sigma}\right) \rightarrow \operatorname{Rep}(G)
$$

denote holomorphic induction. Let $G / G_{\sigma}$ be equipped with the standard complex structure coming from its identification with a coadjoint orbit $G \cdot \mu$, for any $\mu \in \sigma$. For every almost complex $G_{\sigma}$-orbifold $Y_{\sigma}$, the associated bundle $G \times_{G_{\sigma}} Y_{\sigma}$ has a naturally induced almost complex structure. Given a $G_{\sigma}$-invariant orbifold line bundle $L_{\sigma} \rightarrow Y_{\sigma}$, one has

$$
\operatorname{RR}\left(G \times_{G_{\sigma}} Y_{\sigma}, G \times_{G_{\sigma}} L_{\sigma}\right)=\operatorname{Ind}_{G_{\sigma}}^{G} \operatorname{RR}\left(Y_{\sigma}, L_{\sigma}\right) .
$$

For a proof, see e.g. [36].

If $M$ is a Hamiltonian $G$-orbifold with moment map $\Phi: M \rightarrow \mathfrak{g}^{*}$, and $L \rightarrow M$ a $G$-equivariant line bundle, one can always choose a $G$-invariant compatible almost complex structure $J$ on $M$ to define $\mathrm{RR}(M, L)$. Since any two $J$ 's are equivariantly homotopic, this definition does not depend on the choice of $J$. We will be mostly interested in the case that $L$ is a pre-quantum line bundle. In this case we often drop the line bundle from the notation and write $\operatorname{RR}(M):=\mathrm{RR}(M, L)$. 


\subsection{Desingularization for quotients by compact groups}

In this section we define Riemann-Roch numbers for singular symplectic quotients by compact groups and state the "quantization commutes with reduction" Theorem, referring to [37] for more details.

Suppose that $M$ is a Hamiltonian $H \times G$-orbifold ( $H, G$ compact), such that the $G$-action has proper moment map $\Phi: M \rightarrow \mathfrak{g}^{*}$, and let $L \rightarrow M$ be a $H \times G$-equivariant pre-quantum bundle. Let $M_{0}=M / / G$ be the symplectic quotient by $G$ and $L / / G=L_{0}:=\left(L \mid \Phi^{-1}(0)\right) / G$ the quotient bundle.

Following [37] let us call $\alpha \in \mathfrak{g}^{*}$ a quasi-regular value of $\Phi$ if the dimension of the isotropy group $G_{x}$ does not jump as $x$ varies in the level set $\Phi^{-1}(\alpha)$. It can be shown ([37]) that if 0 is a quasi-regular value then $M_{0}$ is a symplectic orbifold and $L_{0}$ with its induced connection is an orbifold pre-quantum line bundle. If 0 is not a quasi-regular value so that the reduced space $M_{0}$ has more serious singularities, one can define $\mathrm{RR}\left(M_{0}\right)$ as the $H$-equivariant Riemann-Roch number of an orbifold line bundle $\tilde{L}_{0} \rightarrow \tilde{M}_{0}$ obtained from $L_{0} \rightarrow M_{0}$ by means of partial desingularization. One desingularization procedure is due to Kirwan [27]. Roughly speaking, this is an inductive procedure involving a sequence of symplectic blow-ups on $M$, resulting in a new Hamiltonian $H \times G$-orbifold $\tilde{M}$, followed by a symplectic quotient $\tilde{M}_{0}=\tilde{M} / / G$. We take $\operatorname{RR}\left(\tilde{M}_{0}, \tilde{L}_{0}\right)$ as the definition of $\operatorname{RR}\left(M_{0}\right)$ (see [37] for details).

A simpler, but less canonical way of desingularizing a symplectic quotient is to shift the value of the moment map. For quasi-regular values $\alpha \in \Phi(M)$ close to zero one can take $\left(L \mid \Phi^{-1}(\alpha)\right) / G_{\alpha} \rightarrow M_{\alpha}$ as a desingularization of $L_{0} \rightarrow M_{0}$. It is shown in [37] that if $\alpha \in \Phi(M)$ is sufficiently close to 0 , then $R R\left(M_{\alpha},\left(L \mid \Phi^{-1}(\alpha)\right) / G_{\alpha}\right)=\operatorname{RR}\left(M_{0}\right)$.

Theorem 5.1 (Quantization commutes with reduction). [36, 37] Let $(M, \omega)$ be a compact Hamiltonian $G \times H$-orbifold with $G \times H$ equivariant pre-quantum line bundle $L \rightarrow M$. Then the $G$-invariant part of $R R(M)$ equals the Riemann-Roch number of the symplectic quotient:

$$
\mathrm{RR}(M)^{G}=\mathrm{RR}(M / / G),
$$

where the right-hand side is defined via partial desingularization if 0 is not a regular value of the $G$-moment map.

If $\mu \in \Lambda_{+}^{*}$ is a dominant weight, the coadjoint orbit $\mathcal{O}_{\mu}=G \cdot \mu$ with its KKS form has a pre-quantum line bundle $\Xi\left(\mathcal{O}_{\mu}\right) \rightarrow \mathcal{O}_{\mu}$. Given a $G$-equivariant pre-quantum line bundle $L \rightarrow M$ as above, we define the 
reduced pre-quantum line bundle by

$$
L_{\mu}:=\left(L \otimes \Xi\left(\mathcal{O}_{* \mu}\right)\right) / / G \rightarrow M_{\mu}=\left(M \times \mathcal{O}_{* \mu}\right) / / G .
$$

As a direct corollary to Theorem 5.1, the multiplicity $N(\mu)$ for the weight $\mu$ to occur in $\operatorname{RR}(M)$ is given by $\operatorname{RR}\left(M_{\mu}\right)$, defined by canonical desingularization if necessary.

\subsection{Desingularizations for loop group quotients}

We now define desingularizations for reductions by loop group actions. Let $G$ be a compact connected simply-connected Lie group, and $M$ a Hamiltonian $L(G \times G)$-manifold with proper moment map at level $m$ and $L(G \times G)$-equivariant pre-quantum line bundle $L$. We will show that the reduction $L / / L G \rightarrow M / / L G$ by the diagonal action can be written in a canonical way as a reduction in finite dimensions, so the desingularization of $L / / L G$ can be carried out as in the previous section.

Let $\mathcal{M}^{m}\left(\Sigma_{0}^{3}\right)$ denote the moduli space for the three-holed sphere at level $m, L^{m}\left(\Sigma_{0}^{3}\right)$ its pre-quantum line bundle and

$$
M^{(1)}=M \times \mathcal{M}^{m}\left(\Sigma_{0}^{3}\right) / / L G \times L G, \quad L^{(1)}=L \times L^{m}\left(\Sigma_{0}^{3}\right) / / L G \times L G
$$

the quotient by the product of the two diagonal $L G$-actions, defined by pairing each $L G$-factor for $M$ with an $L G$-factor for $\mathcal{M}^{m}\left(\Sigma_{0}^{3}\right)$. Since these actions are free, $M^{(1)}$ is a Hamiltonian $L G$-manifold and $L^{(1)}$ an $\widehat{L G}$-equivariant pre-quantum line bundle. Because the reduction of $\mathcal{M}^{m}\left(\Sigma_{0}^{3}\right)$ at 0 is the moduli space $\mathcal{M}^{m}\left(\Sigma_{0}^{2}\right)$, the reduction of $M^{(1)}$ at 0 is given by

$$
M_{0}^{(1)}=\left(M \times \mathcal{M}^{m}\left(\Sigma_{0}^{2}\right) / / L G\right) / / L G=M / / L G
$$

by Example 4.2. Let $Y_{\{0\}}^{(1)}$ be the symplectic cross-section for $M^{(1)}$ at $\{0\} \subset \mathfrak{A}$. Then

$$
M / / L G \cong M_{0}^{(1)} \cong Y_{\{0\}}^{(1)} / / G, \quad L / / L G \cong L_{0}^{(1)} \cong L_{0}^{(1)} \mid Y_{\{0\}}^{(1)} / / G
$$

which proves the claim. In particular, the Riemann-Roch numbers of the line bundles in (25) can be defined by desingularization. Note that in the case of moduli spaces, the above procedure corresponds to "adding a puncture" to the 2-manifold. 


\section{Symplectic surgery}

In this section, we apply Lerman's symplectic cutting procedure together with Proposition 4.9 to prove the factorization property Theorem 2.1 .

\subsection{Symplectic cutting}

We briefly recall Lerman's cutting construction [32]. Let $M$ be a Hamiltonian $S^{1}$-orbifold, with moment map $\psi: M \rightarrow \mathbb{R}$. Consider the action of $S^{1}$ on the product $M \times \mathbb{C}$ given by $e^{2 \pi i t} \cdot(m, z)=\left(e^{2 \pi i t} \cdot m, e^{2 \pi i t} z\right)$, with moment map

$$
\tilde{\psi}(m, z)=\psi(m)-\pi|z|^{2} .
$$

The zero level set of $\tilde{\psi}$ is a union of $\psi^{-1}(0) \times\{0\}$ and the set of all $(m, z)$ with $\psi(m)=|z|^{2}>0$. Suppose that 0 is a regular value of $\psi$. Then 0 is also a regular value of $\tilde{\psi}$, and the reduced space $M_{+}:=M \times \mathbb{C} / / S^{1}$ is a symplectic orbifold. As a topological space, $M_{+}$is obtained from the manifold with boundary $\psi^{-1}\left(\mathbb{R}_{\geq 0}\right)$ by collapsing the boundary by the nullfoliation of the pullback of the symplectic form.

It was shown by Lerman that this is also true symplectically:

Proposition 6.1 (Lerman). Let

$$
M_{+}:=(M \times \mathbb{C}) / / S^{1}
$$

be the cut space. The canonical maps

$$
\iota_{0}: M_{0} \rightarrow M_{+}, \quad \iota_{>0}: \psi^{-1}\left(\mathbb{R}_{>0}\right) \rightarrow M_{+}
$$

are smooth symplectic embeddings, and the normal bundle of $M_{0}$ in $M_{+}$ is canonically isomorphic to the associated bundle $\psi^{-1}(0) \times{ }_{S^{1}} \mathbb{C}$, where $S^{1}$ acts on $\mathbb{C}$ with weight 1 . Given a Hamiltonian action of a Lie group $G$ on $M$, with moment map $\Phi: M \rightarrow \mathfrak{g}^{*}$, such that this action commutes with the action of $S^{1}$, there is a naturally induced Hamiltonian $G$-action on $M_{+}$, which agrees with the given actions and moment maps on $M_{0}$ and $\psi^{-1}\left(\mathbb{R}_{>0}\right)$.

By reversing the $S^{1}$-action, one can also define a cut-space $M_{-}$, which is the union of $M_{0}$ and $\psi^{-1}\left(\mathbb{R}_{<0}\right)$.

The orbifold structure on $M_{ \pm}$depends only on the circle action on $\psi^{-1}(0)$. Replacing the given circle by a covering introduces additional orbifold singularities. 
Suppose that we are given an $S^{1} \times G$-equivariant complex vector bundle $E \rightarrow M$. Let $E_{0} \rightarrow M_{0}$ be the reduced bundle $E_{0}=E \mid \psi^{-1}(0) / S^{1}$. By pulling $E$ back to $M \times \mathbb{C}$, restricting to $\tilde{\psi}^{-1}(0)$ and taking the quotient

$$
E_{+}:=\left(\operatorname{pr}_{1}^{*} E \mid \tilde{\psi}^{-1}(0)\right) / S^{1},
$$

we obtain a $G$-vector bundle over $M_{+}$. There are canonical equivariant isomorphisms

$$
\iota_{0}^{*} E_{+} \cong E_{0}, \iota_{>0}^{*} E_{+} \cong E \mid \psi^{-1}\left(\mathbb{R}_{>0}\right)
$$

If $E=L$ is a pre-quantum line bundle, then $L_{+}=(L \otimes \mathbb{C}) / / S^{1}$ with induced metric and connection is a pre-quantum line bundle for $M_{+}$.

Cutting is a local operation. That is, if $M$ is a Hamiltonian $G$-space the cut spaces $M_{ \pm}$are defined for any separating $G$-invariant hypersurface $Z \subset M$, together with a $G$-equivariant locally free Hamiltonian circle action on some neighborhood of $Z$ and having $Z$ as its zero level set $\psi^{-1}(0)$. Likewise, given a $G$-equivariant vector bundle $E \rightarrow M$ together with a $G$-equivariant lift of the $S^{1}$-action one obtains $G$-equivariant "cut bundles" $E_{+} \rightarrow M_{+}$and $E_{-} \rightarrow M_{-}$. Assume that $M$ is compact and that $E=L$ is a line bundle.

Proposition 6.2 [36]. The Riemann-Roch numbers of the cut bundles satisfy the gluing rule

$$
\operatorname{RR}(M, L)=\operatorname{RR}\left(M_{+}, L_{+}\right)+\operatorname{RR}\left(M_{-}, L_{-}\right)-\operatorname{RR}\left(M_{0}, L_{0}\right) .
$$

Let us briefly recall the proof from [36] for the simplest case that $G$ is trivial, $M$ is a manifold, and the $S^{1}$-action on $Z$ is free. Let $U \cong Z \times[-\epsilon, \epsilon]$ be a tubular neighborhood of $Z$ on which the $S^{1}$-action is defined. By the index theorem, $\operatorname{RR}(M, L)$ is given by an integral

$$
\begin{aligned}
\operatorname{RR}(M, L) & =\int_{M} \operatorname{Td}(M) \operatorname{Ch}(L) \\
& =\int_{U} \operatorname{Td}(M) \operatorname{Ch}(L)+\int_{M \backslash U} \operatorname{Td}(M) \operatorname{Ch}(L),
\end{aligned}
$$

where $\operatorname{Td}(M), \operatorname{Ch}(L) \in \Omega^{*}(M)$ are the Todd form and Chern character form, constructed using connections on the bundles $T M$ and $L$. We choose the almost complex structure on $T M$ and the connections on $T M$ and $L$ to be $S^{1}$-invariant over $U$. Then the Todd and Chern 
forms have extensions to $S^{1}$-equivariant characteristic forms (cf. [8]) $\operatorname{Td}(U, \xi), \operatorname{Ch}(U, \xi)$ for $\xi$ sufficiently small, so that

$$
\int_{U} \operatorname{Td}(M) \operatorname{Ch}(L)=\left.\left(\int_{U} \operatorname{Td}(U, \xi) \operatorname{Ch}\left(\left.L\right|_{U}, \xi\right)\right)\right|_{\xi=0} .
$$

The last integral can be re-written, using the Berline-Vergne localization formula [8] for manifolds with boundary, as an equality of rational functions for small $\xi$,

$$
\int_{U} \operatorname{Td}(U, \xi) \operatorname{Ch}\left(\left.L\right|_{U}, \xi\right)=\int_{\partial U} \operatorname{Td}(U, \xi) \operatorname{Ch}\left(\left.L\right|_{U}, \xi\right) \frac{\alpha}{\mathrm{d} \alpha-\xi}
$$

where $\alpha$ is a connection 1-form on $\partial U$. Consider on the other hand the index formula for $\operatorname{RR}\left(M_{ \pm}, L_{ \pm}\right)$. Let $\mathrm{Td}\left(M_{ \pm}\right)$and $\mathrm{Ch}\left(L_{ \pm}\right)$be the Todd and Chern character form for the cut spaces. We may choose the almost complex structures and connections entering their definitions to agree with those for $M$ outside $Z \times[-\epsilon / 2, \epsilon / 2]$. By the index theorem applied to $M_{ \pm}$we may write $\operatorname{RR}\left(M_{ \pm}, L_{ \pm}\right)$as a sum of integrals over $M_{ \pm} \backslash U_{ \pm}$ and $U_{ \pm}$. By the localization theorem applied to $U_{ \pm}$we have

$$
\begin{aligned}
\int_{U_{ \pm}} \operatorname{Td}\left(U_{ \pm}, \xi\right) \operatorname{Ch}\left(\left.L\right|_{U_{ \pm}}, \xi\right)= & \int_{\partial U_{ \pm}} \operatorname{Td}(U, \xi) \operatorname{Ch}\left(\left.L\right|_{U}, \xi\right) \frac{\alpha}{\operatorname{d} \alpha-\xi} \\
& +\int_{M_{0}} \frac{\operatorname{Td}\left(M_{ \pm}\right) \operatorname{Ch}\left(L_{ \pm}\right)}{\operatorname{Eul}\left(\nu_{ \pm}, \xi\right)}
\end{aligned}
$$

so that

$$
\begin{aligned}
\operatorname{RR}\left(M_{+}, L_{+}\right)+\mathrm{RR}\left(M_{-}, L_{-}\right)-\mathrm{RR}(M, L) \\
=\int_{M_{0}}\left(\frac{\operatorname{Td}\left(M_{+}\right) \mathrm{Ch}\left(L_{+}\right)}{\operatorname{Eul}\left(\nu_{+}, \xi\right)}+\frac{\operatorname{Td}\left(M_{-}\right) \operatorname{Ch}\left(L_{-}\right)}{\operatorname{Eul}\left(\nu_{-}, \xi\right)}\right) .
\end{aligned}
$$

Since $T M_{ \pm}=T M_{0} \oplus \nu_{ \pm}$and

$$
\operatorname{Td}\left(\nu_{ \pm}, \xi\right)=\frac{\operatorname{Eul}\left(\nu_{ \pm}, \xi\right)}{1-\operatorname{Ch}\left(\nu_{ \pm}^{*}, \xi\right)}
$$

the additional terms may be re-written

$$
\int_{M_{0}} \operatorname{Td}\left(M_{0}\right) \operatorname{Ch}\left(L_{0}\right)\left(\frac{1}{1-\operatorname{Ch}\left(\nu_{+}^{*}, \xi\right)}+\frac{1}{1-\operatorname{Ch}\left(\nu_{-}^{*}, \xi\right)}\right) .
$$

Because $\nu_{+}=\nu_{-}^{*}$, the identity $1 /(1-z)+1 /\left(1-z^{-1}\right)=1$ implies that

$$
\frac{1}{1-\operatorname{Ch}\left(\nu_{+}^{*}, \xi\right)}+\frac{1}{1-\operatorname{Ch}\left(\nu_{-}^{*}, \xi\right)}=1
$$

which completes the proof. 


\subsection{Factorization in rank-one}

In this section, we prove Theorem 2.1 in the simplest case in which $G=S U(2)$ and 0 is a regular value for the diagonal action. We identify the Cartan algebra $\mathfrak{t} \cong \mathbb{R}$ so that the weight lattice $\Lambda^{*} \cong \mathbb{Z}$ and the fundamental alcove $\mathfrak{A}=[0,1]$. Let $M$ be a Hamiltonian $L(G \times G)$ manifold with proper moment map at level $m \in \mathbb{N}$ and pre-quantum line bundle $L \rightarrow M$, and $L / / L G \rightarrow M / / L G$ the symplectic reduction with respect to the diagonal $L G$-action. Since 0 is a regular value for the diagonal action, $M / / L G$ is a compact symplectic orbifold, and $L / / L G$ a pre-quantum line bundle. Let $\phi: M / / L G \rightarrow \mathfrak{A}$ denote the residual toric moment map, and $\nu \in(0,1) \cap \mathbb{Q}$ a regular value of $\phi$. Let $r \in \mathbb{Z}_{+}$ be such that $r \nu \in \mathbb{Z}$. Let $\psi: M / / L G \rightarrow \mathbb{R}$ denote the map

$$
\psi=r(\phi-\nu)
$$

so that $\psi$ is a moment map for the $r$-fold covering of the residual $S^{1}$ action. One obtains a pre-quantum action of $S^{1}$ on $L / / L G$ by multiplying the residual action by the character corresponding to the weight $-r m \nu$.

By the Gluing Formula, Proposition 6.2 we obtain

$$
\begin{aligned}
\mathrm{RR}(M / / L G)= & \mathrm{RR}\left((M / / L G)_{+}\right)+\mathrm{RR}\left((M / / L G)_{-}\right) \\
& -\operatorname{RR}\left((M / / L G)_{0}\right) .
\end{aligned}
$$

The cut space $(M / / L G)_{-}$can be written as a reduction in finite dimensions: Let $\sigma=\{0\}$ and $Y_{\sigma,-\sigma}$ denote the corresponding cross-section of $M$. The restriction to $Y_{\sigma,-\sigma}$ of $\tilde{\Phi}_{+}$defines a circle action on a dense subset of $Y_{\sigma,-\sigma}$, and as above one can cut using the function $r\left(\tilde{\Phi}_{+}-\nu\right)$. Let $\left(Y_{\sigma,-\sigma}\right)_{-}$denote the negative part of the cut space. Since the $S^{1}$ and $G$-actions commute, the operation of cutting commutes with reduction and by Theorem 4.9 we are led to

$$
(M / / L G)_{-}=\left(Y_{\sigma,-\sigma}\right)_{-} / / G .
$$

The space $\left(Y_{\sigma,-\sigma}\right)_{-}$may not be compact, but may be replaced with a compact space by another cutting operation using $\tilde{\Phi}_{+}$. By Theorem 5.1 we get

$$
\mathrm{RR}\left((M / / L G)_{-}\right)=\sum_{\mu \in \Lambda_{m}^{*}} \operatorname{RR}\left(\left(\left(Y_{\sigma,-\sigma}\right)_{-}\right)_{\mu, * \mu}\right)=\sum_{\mu \in \Lambda_{m}^{*}, \mu \leq m \nu} \operatorname{RR}\left(M_{\mu, * \mu}\right) .
$$

Similarly, $\operatorname{RR}\left((M / / L G)_{+}\right)$is the sum of $\operatorname{RR}\left(M_{\mu, * \mu}\right)$ over $\mu \geq m \nu$, and $\operatorname{RR}\left((M / / L G)_{0}\right)=\operatorname{RR}\left(M_{m \nu, * m \nu}\right)$, so the Theorem is proved by $(29)$. 


\subsection{Multiple cuts}

In order to deal with higher rank groups one needs the following generalization of symplectic cutting. Let $M$ be a compact symplectic orbifold, $T$ a torus with Lie algebra $\mathfrak{t}$. Let $\psi: M \rightarrow \mathfrak{t}^{*}$ be a continuous map. Let $Q \subset \mathfrak{t}^{*}$ be a rational simplicial polytope. For any face $F \subset Q$, let $\mathfrak{t}_{F} \subseteq \mathfrak{t}$ be the annihilator of the affine space spanned by $F$, and $T_{F}$ the torus with Lie algebra $\mathfrak{t}_{F}$. Let $\pi_{F}: \mathfrak{t}^{*} \rightarrow \mathfrak{t}_{F}^{*}$ denote the projection.

Definition 6.3. The polytope $Q$ is called $\psi$-admissible if for every open face $F \subset Q$, the composition $\pi_{F} \circ \psi$ is a smooth moment map for a locally free action of $T_{F}$ on a neighborhood $U_{F}$ of $\psi^{-1}(F)$.

The definition implies that if $Q$ is $\psi$-admissible and $F \subset Q$ an open face, then the pre-image $\psi^{-1}(F)$ is a smooth coisotropic suborbifold, with null-foliation given by the $T_{F}$-orbits. Hence the quotient

$$
M_{F}:=\psi^{-1}(F) / T_{F}
$$

is a symplectic orbifold. We define the cut space $M_{Q}$ of $M$ as a union

$$
M_{Q}=\coprod_{F \subset Q} M_{F}
$$

Thus $M_{Q}$ is obtained from the orbifold with corners $\psi^{-1}(Q)$ by collapsing the boundary faces under the $T_{F}$-actions. An orbifold structure on $M_{Q}$ is given as follows: Let $F_{1}, \ldots, F_{l}$ be the facets (codimension 1 faces) of $Q$ containing $F$. The open subset $\left(U_{F}\right)_{Q} \subset M_{Q}$ is formed by symplectic reduction of $U_{F}$ by $T_{Q}$, followed by iterated symplectic cuts by the circles $T_{F_{1}} / T_{Q}, \ldots, T_{F_{l}} / T_{Q}$.

If $M$ is a Hamiltonian $G$-orbifold and $\psi$ is $G$-invariant, then the cut spaces $M_{Q}$ are Hamiltonian $G$-orbifolds.

Definition 6.4. Given $M$ and $\psi$ as above, a $\psi$-admissible polyhedral subdivision of $t^{*}$ is a collection $\mathcal{Q}$ of rational, simplicial polytopes satisfying the following conditions: every element of $\mathcal{Q}$ is $\psi$-admissible, their union is $\mathfrak{t}^{*}$, for every element in $\mathcal{Q}$ all its closed faces are also in $\mathcal{Q}$, and the intersection of any two elements of $\mathcal{Q}$ is a face of each.

We have three examples in mind.

a. Let $M$ be a compact Hamiltonian $G$-orbifold, and $\psi: M \rightarrow \mathfrak{t}_{+}^{*}$ the induced toric moment map as in (18). Recall that the dual cone $C_{\sigma}$ to $\mathfrak{t}_{+}^{*}$ at an open face $\sigma \subset \mathfrak{t}_{+}^{*}$ is defined to be the set of all 
vectors $v \in \mathfrak{t}^{*}$ such that the minimum of the inner product $(v, x)$ for $x \in \mathfrak{t}_{+}^{*}$ is achieved by $x \in \sigma$. (Here we use an inner product on $\mathfrak{t}$ coming from an invariant inner product on $\mathfrak{g}$; the definitions are independent of this choice.) Let $\mu \in \operatorname{int}\left(t_{+}^{*}\right)$. For each face $\tau \subseteq \bar{\sigma}$ let $Q_{\tau, \sigma}$ denote the polytope $\mu+\bar{\tau}-C_{\sigma}$. The faces of $Q_{\tau, \sigma}$ are all polytopes $Q_{\tau^{\prime}, \sigma^{\prime}}$ such that $\tau^{\prime} \subseteq \bar{\tau}$ and $\sigma \subseteq \overline{\sigma^{\prime}}$. Moreover if $\tau_{1} \subseteq \bar{\sigma}_{1}$ and $\tau_{2} \subseteq \bar{\sigma}_{2}$ one has

$$
Q_{\tau_{1}, \sigma_{1}} \cap Q_{\tau_{2}, \sigma_{2}}=Q_{\tau_{1} \wedge \tau_{2}, \sigma_{1} \vee \sigma_{2}},
$$

where $\tau_{1} \wedge \tau_{2}$ is the largest open face contained in $\overline{\tau_{1}} \cap \overline{\tau_{2}}$, and $\sigma_{1} \vee \sigma_{2}$ is the smallest open face whose closure contains $\sigma_{1} \cup \sigma_{2}$. Let $\mathcal{Q}=\left\{Q_{\tau, \sigma} \mid \tau \subseteq \bar{\sigma}\right\}$ (see Figure 2). For generic choices of $\mu$, the collection $\mathcal{Q}$ of these polytopes is a $\psi$-admissible polyhedral subdivision (c.f. Remark 2.3 in [33]).

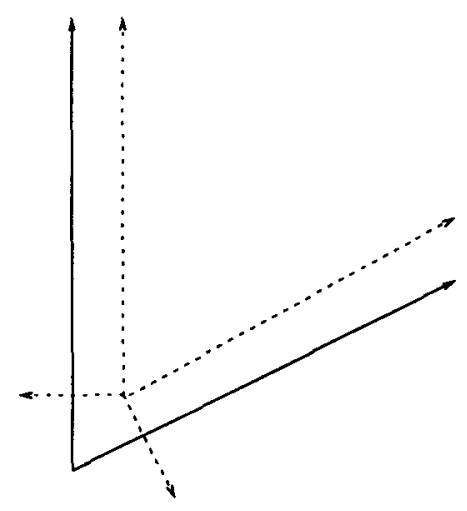

Figure 2. The collection $\mathcal{Q}$ for $G=S U(3)$

b. Consider the case that $N$ is a compact Hamiltonian $G \times G$-orbifold, with moment map $\left(\Phi_{+}, \Phi_{-}\right)$and assume that 0 is a regular value for the diagonal $G$-action. Let $M=N / / G$ be the reduced space and $\psi: M \rightarrow t_{+}^{*}$ the residual toric moment map induced from the map $\tilde{\Phi}_{+}=q \circ \Phi_{+}$on the first factor. For generic choices of $\mu$, the collection $\mathcal{Q}$ described above is a $\psi$-admissible polyhedral subdivision.

c. Finally, let $G$ be a compact, connected, simply-connected Lie group, and $\mathfrak{A}$ the corresponding fundamental alcove. Let $N$ be 
a Hamiltonian $L(G \times G)$-manifold with proper moment map $\Phi=$ $\left(\Phi_{+}, \Phi_{-}\right)$(at level $\left.\lambda=+1\right)$. We assume that the diagonal $L G$ action is locally free on its zero level set, so that the symplectic quotient $M=N / / L G$ is a finite dimensional, compact symplectic orbifold. Let $\psi: M \rightarrow \mathfrak{A}$ denote the residual toric moment map induced from $q \circ \Phi_{+}$. For each face $\sigma \subset \mathfrak{A}$, the open set $\psi^{-1}\left(U_{\sigma}\right) \subseteq M$ carries a Hamiltonian action of the center $Z\left((L G)_{\sigma}\right)$, with moment map given by $\psi$ followed by projection to $\mathfrak{z}\left((L \mathfrak{g})_{\sigma}\right)^{*}$.

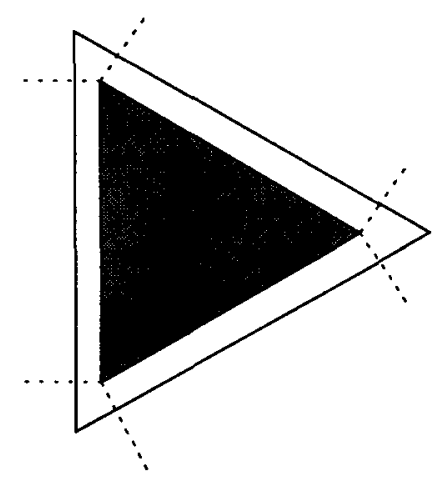

FIgURE 3. Cutting the fundamental alcove for $G=S U(3)$

The construction of the polyhedral subdivision depends on choices of $\epsilon \in \mathbb{R}_{>0}$ and $\mu \in \operatorname{int}(\mathfrak{A})$. For any pair of faces $\sigma, \tau$ with $\tau \subseteq \bar{\sigma}$, let

$$
Q_{\tau, \sigma}=(1-\epsilon) \bar{\tau}+\epsilon \mu-C_{\sigma} .
$$

For example the shaded region in Figure 3 is $Q_{\tau, \tau}$ where $\tau=$ $\operatorname{int}(\mathfrak{A})$. Let $\mathcal{Q}=\left\{Q_{\tau, \sigma} \mid \tau \subseteq \bar{\sigma}\right\}$ (see Figure 3). For generic choices of $\epsilon, \mu$, each $Q \in \mathcal{Q}$ is $\psi$-admissible.

\subsection{Cut bundles}

Let $E \rightarrow M$ be a complex vector bundle, and suppose that on each neighborhood $U_{F}$ we have a lift of the $T_{F}$-action. The cut bundle $E_{Q}$ defined by

$$
E_{Q}=\bigcup_{F \subset Q}\left(E \mid \psi^{-1}(F)\right) / T_{F}
$$


has the structure of a complex orbi-bundle. If $E=L$ is a pre-quantum line bundle, with pre-quantum lifts with respect to the maps $\pi_{F} \circ \psi$, then the bundle $L_{Q}$ is a pre-quantum line bundle over $M_{Q}$.

We now explain how such vector bundles arise in each of our three examples.

a. If $E \rightarrow M$ is a $G$-equivariant vector bundle, then as explained in Section 4.2 there is a canonical lift of the $Z\left(G_{\sigma}\right) \times G$-action on $\psi^{-1}\left(U_{\sigma}\right)=G \cdot Y_{\sigma}$. Therefore one also has a canonical lift of the local torus actions, and a corresponding cut bundle $E_{Q}$. However, if $E=L$ is a $G$-equivariant pre-quantum bundle, then this lift does not in general satisfy the pre-quantum condition, and therefore the cut bundle $L_{Q}$ is not pre-quantum.

However, if $Q$ is a lattice polytope, then $\pi_{F}(F) \in \mathfrak{t}_{F}^{*}$ is a weight. One therefore obtains local pre-quantum lifts by multiplying the given $T_{F}$-actions by the character corresponding to the weight $\pi_{F}(F)$. The cut bundle $L_{Q}$ for the modified actions is a $G$ equivariant pre-quantum line bundle. In case $Q$ has rational vertices, choose any covering $\tilde{T} \rightarrow T$ such that $Q$ is a lattice polytope in the refined lattice $\tilde{\Lambda}^{*} \supset \Lambda^{*}$. The local actions of the corresponding covers $\tilde{T_{F}} \rightarrow T_{F}$ admit pre-quantum lifts as before. Notice again that passing to a cover introduces additional orbifold singularities.

b. If $E \rightarrow M$ is a $G \times G$-equivariant vector bundle, the quotient bundle $E_{0}$ has a canonical lift of the local $Z\left(G_{\sigma}\right)$-actions on $M_{0}$, so that there is a canonical cut bundle. The same argument as above shows that if $E=L$ is a pre-quantum line bundle and $Q$ is a rational polytope, one can modify the given lift of the local actions in such a way that the cut bundle is a pre-quantum line bundle.

c. For every $\widehat{L G^{2}}$-equivariant vector bundle $E \rightarrow M$, any lift of the local $Z\left((L G)_{\sigma}\right)$-actions to $E_{0}=E / / K$ defines a cut-bundle $\left(E_{0}\right)_{Q}$. If $L \rightarrow M$ is a $\widehat{L G^{2}}$-equivariant pre-quantum line bundle, and $\epsilon \in \mathbb{Q}, \mu \in \Lambda^{*} \otimes_{\mathbb{Z}} \mathbb{Q}$ are chosen so that each $Q$ is a polytope with rational vertices, one obtains by the same construction as in the previous two examples a pre-quantum cut-bundle $\left(L_{0}\right)_{Q}$. 


\subsection{The gluing formula}

The following Theorem shows how the Riemann-Roch number of the original bundle decomposes into Riemann-Roch numbers for cut bundles.

Theorem 6.5 (Gluing Formula). Let $M$ be a compact Hamiltonian $G$-orbifold, and $L \rightarrow M$ a G-equivariant line bundle. Given a map $\psi: M \rightarrow \mathfrak{t}^{*}$, a $\psi$-admissible polyhedral subdivision $\mathcal{Q}$, and lifts to $L$ of the local torus actions defined by $\psi$, the Riemann-Roch number of $L$ is given by

$$
\mathrm{RR}(M, L)=\sum_{Q \in \mathcal{Q}}(-1)^{\operatorname{codim}(Q)} \mathrm{RR}\left(M_{Q}, L_{Q}\right)
$$

For collections $\mathcal{Q}$ of polytopes constructed from induced toric maps for Hamiltonian actions of compact groups $G$ (that is, for the first example given in Section 6.3) the above gluing formula was proved in [36]. In the appendix we outline the proof of Theorem 6.5 for the more general situation.

As an application we prove:

Proposition 6.6. Let $G$ be a compact connected Lie group and $M$ a Hamiltonian $G \times G$-orbifold with proper moment map $\Phi=\left(\Phi_{+}, \Phi_{-}\right)$. Let $L \rightarrow M$ be a $G \times G$-equivariant pre-quantum line bundle. If the symplectic quotient $M_{0}=M / / G$ by the diagonal action is compact, the Riemann-Roch number of the reduced bundle $L_{0}=L / / G$ is given by

$$
\operatorname{RR}\left(M_{0}\right)=\sum_{\mu \in \Lambda_{+}^{*}} \operatorname{RR}\left(M_{\mu, * \mu}\right) .
$$

Here all RR-numbers are defined by partial desingularization if necessary.

Proof. If $M$ is compact, both sides are equal, by the "quantization commutes with reduction" Theorem 5.1. If $M$ is not compact, let $\tilde{\Phi}_{0}$ : $M_{0} \rightarrow \mathfrak{t}^{*}$ denote the residual toric moment map induced by $\tilde{\Phi}_{+} \cdot$ Its image is given by

$$
\tilde{\Phi}_{0}\left(M_{0}\right)=\tilde{\Phi}_{+}(M) \cap * \tilde{\Phi}_{-}(M) .
$$

Choose a compact admissible rational polytope $Q \subset \mathfrak{t}^{*} \times \mathfrak{t}^{*}$, such that $\tilde{\Phi}_{0}\left(M_{0}\right) \times * \tilde{\Phi}_{0}\left(M_{0}\right)$ is contained in the relative interior of $Q \cap\left(\mathfrak{t}_{+}^{*} \times \mathfrak{t}_{+}^{*}\right)$. We can assume that $Q$ has rational vertices, so that the cut bundle 
$L_{Q} \rightarrow M_{Q}$ is a $G$-equivariant pre-quantum bundle. Since $M_{Q}$ is compact and $L / / G=L_{Q} / / G$, this reduces the proof to the case where $M$ is compact. q.e.d.

\subsection{Proof of factorization in arbitrary rank}

As in the statement of Theorem 2.1, let $G$ be compact, connected and simply connected, and $\mathfrak{A}$ its fundamental alcove. Let $M$ be a Hamiltonian $L(G \times G)$-manifold with proper moment map at level $m \in \mathbb{N}$ and pre-quantum line bundle $L \rightarrow M$, and $L / / L G \rightarrow M / / L G$ the symplectic reduction with respect to the diagonal $L G$-action.

Let us first assume that 0 is a regular value for the diagonal action, so that $M / / L G$ is a compact symplectic orbifold, and $L / / L G \rightarrow M / / L G$ a pre-quantum line bundle. Let $\mathcal{Q}$ be a collection of admissible rational polytopes in $\mathfrak{A}$ defined in the previous subsection. By the Gluing Formula, Equation (30) we get

$$
\operatorname{RR}(M / / L G)=\sum_{Q \in \mathcal{Q}}(-1)^{\operatorname{codim}(Q)} \operatorname{RR}\left((M / / L G)_{Q}\right)
$$

Each cut space $(M / / L G)_{Q}$ can be written as a reduction in finite dimensions: Let $\sigma \subset \mathfrak{A}$ be an open face such that $Q \cap \mathfrak{A}$ is contained in $U_{\sigma}$, and let $Y_{\sigma,-\sigma}$ denote the corresponding cross-section of $M$. The restriction to $Y_{\sigma,-\sigma}$ of $\tilde{\Phi}_{+}$defines an induced toric action on $Y_{\sigma,-\sigma}$; let $\left(Y_{\sigma,-\sigma}\right)_{Q}$ be the corresponding cut space. Then Proposition 4.9, implies

$$
(M / / L G)_{Q}=\left(Y_{\sigma,-\sigma}\right)_{Q} / / K_{\sigma}, \quad(L / / L G)_{Q}=\left(L \mid Y_{\sigma,-\sigma}\right)_{Q} / / K_{\sigma} .
$$

By Proposition 6.6 applied to $\left(L \mid Y_{\sigma,-\sigma}\right)_{Q}$ we obtain that

$$
\operatorname{RR}\left((M / / L G)_{Q}\right)=\sum_{\mu \in Q \cap \Lambda_{m}^{*}} \operatorname{RR}\left(M_{\mu, * \mu}\right) .
$$

Finally, applying the Euler identity $\sum_{Q \ni \mu}(-1)^{\operatorname{codim}(Q)}=1$ yields

$$
\begin{aligned}
\operatorname{RR}(M / / L G) & =\sum_{Q \in \mathcal{Q}}(-1)^{\operatorname{codim}(Q)} \sum_{\mu \in Q \cap \Lambda_{m}^{*}} \operatorname{RR}\left(M_{\mu, * \mu}\right) \\
& =\sum_{\mu \in \Lambda_{m}^{*}} \operatorname{RR}\left(M_{\mu, * \mu}\right)
\end{aligned}
$$

which proves the result in this case. 
In the case where 0 is not a regular value, we use a desingularization as explained in section 5.3. Let

$$
M^{(2)}:=M \times \mathcal{M}\left(\Sigma_{0}^{3}\right) / / L G
$$

and

$$
M^{(1)}=M^{(2)} / / L G=M \times \mathcal{M}\left(\Sigma_{0}^{3}\right) / / L G \times L G,
$$

equipped with their pre-quantum bundles. Correspondingly, we will use cross-sections $Y_{\{0\}}^{(1)}$ for $M^{(1)}, Y_{\{0\}, \sigma,-\sigma}^{(2)}$ for $M^{(2)}$, and $Y_{\sigma,-\sigma}$ for $M$. To compactify $Y_{\{0\}}^{(1)}$, we choose an admissible polytope $Q_{1}$ with rational vertices with $0 \in \operatorname{int}\left(Q_{1}\right)$, and use the cut space $\left(Y_{\{0\}}^{(1)}\right)_{Q_{1}}$. We also choose an admissible family of polytopes $\mathcal{Q}_{2}$ to cut $Y_{\sigma,-\sigma}$ and $\left(Y_{\{0\}, \sigma,-\sigma}^{(2)}\right)_{Q_{1}}$ into pieces. Then

$$
\begin{aligned}
& \left(Y_{\{0\}, \sigma,-\sigma}^{(2)}\right)_{Q_{1} \times Q_{2}} / / G \cong\left(Y_{\sigma,-\sigma}\right)_{Q_{2}}, \\
& \left(Y_{\{0\}, \sigma,-\sigma}^{(2)}\right)_{Q_{1} \times Q_{2}} / /(L G)_{\sigma}=\left(Y_{\{0\}}^{(1)}\right)_{Q_{1} \times Q_{2}} .
\end{aligned}
$$

Using the gluing formula, together with repeated application of "quantization commutes with reduction" we compute

$$
\mathrm{RR}(M / / L G)=\operatorname{RR}\left(\left(Y_{\{0\}}^{(1)}\right)_{Q_{1}} / / G\right)=\operatorname{RR}\left(\left(Y_{\{0\}}^{(1)}\right)_{Q_{1}}\right)^{G}
$$

(Theorem 5.1)

$$
=\sum_{Q_{2} \in \mathcal{Q}_{2}}(-1)^{\operatorname{codim}\left(Q_{2}\right)} \operatorname{RR}\left(\left(Y_{\{0\}}^{(1)}\right)_{Q_{1} \times Q_{2}}\right)^{G}
$$

(Theorem 6.5)

$$
=\sum_{Q_{2} \in \mathcal{Q}_{2}}(-1)^{\operatorname{codim}\left(Q_{2}\right)} \operatorname{RR}\left(\left(Y_{\{0\}, \sigma,-\sigma}^{(2)}\right)_{Q_{1} \times Q_{2}}\right)^{(L G)_{\sigma} \times G}
$$

(Theorem 5.1)

$$
=\sum_{Q_{2} \in \mathcal{Q}_{2}}(-1)^{\operatorname{codim}\left(Q_{2}\right)} \operatorname{RR}\left(\left(Y_{\sigma,-\sigma}\right)_{Q_{2}}\right)^{(L G)_{\sigma}}
$$

(Theorem 5.1) 


$$
=\sum_{Q_{2} \in \mathcal{Q}_{2}}(-1)^{\operatorname{codim}\left(Q_{2}\right)} \sum_{\mu \in Q_{2} \cap \Lambda_{m}^{*}} \operatorname{RR}\left(M_{\mu, * \mu}\right)
$$

(Proposition 6.6)

$=\sum_{\mu \in \Lambda_{m}^{*}} \operatorname{RR}\left(M_{\mu, * \mu}\right)$

(Euler Identity).

\section{Appendix A. Proof of the Gluing Formula}

In this appendix we outline the proof of Theorem 6.5, assuming for simplicity that $M$ and all cut spaces are smooth. The details necessary for the extension to orbifold singularities may be found in [36].

The idea is to decompose $M$ into sub-manifolds with corners on which the cutting torus actions are globally defined. On each piece the differential forms entering the index theorem have equivariant extensions, and one can apply localization to prove the gluing formula locally. To construct the decomposition of $M$ choose a second $\psi$-admissible subdivsion $\mathcal{Q}^{\prime}$ of $\mathfrak{t}^{*}$ and let $\mathcal{P} \subset \mathcal{Q}^{\prime}$ be the top dimensional polytopes. For any $P \in \mathcal{P}$ let $T_{(P)} \subset T$ be the smallest subtorus containing all $T_{F}$, for $F$ an open face of some $Q \in \mathcal{Q}$ such that with $F \cap P \neq \emptyset$. Let $\pi_{(P)}: \mathfrak{t}^{*} \rightarrow \mathfrak{t}_{(P)}^{*}$ be the projection.

We make the following two assumptions.

a. On each $\psi^{-1}(P)$, the composition $\pi_{(P)} \circ \psi$ is a smooth moment map for the action of $T_{(P)}$.

b. For any two elements $P \in \mathcal{P}$ and $Q \in \mathcal{Q}$, the intersection $P \cap$ $Q$ is $\psi$-admissible and any pair of faces $F \subset P, H \subset Q$ meet transversally.

The first condition implies that for $Q \in \mathcal{Q}$ and all open faces $F \subset Q$ such that $F \cap P \neq \emptyset$, one has $\psi^{-1}(P) \subset U_{F}$, i.e., the $T_{F}$-actions extend to a global $T_{(P)}$-action on $\psi^{-1}(P)$. The meaning of the second condition is that $\mathcal{Q}$ is an admissible subdivision for the function $\psi_{P}: M_{P} \rightarrow$ $t^{*}$ induced by $\psi$ on the cut space. It is easy to write down explicit subdivisions with these properties in the three examples discussed in Section 6.3. For instance, in the third example one may take $\mathcal{P}$ to be the set of polytopes of maximal dimension in a collection $\mathcal{Q}^{\prime}$ constructed using any smaller value of $\epsilon$. 
Lemma A.7. The Riemann-Roch number $\mathrm{RR}(M, L)$ satisfies the gluing rule

$$
\operatorname{RR}(M, L)=\sum_{P \in \mathcal{P}} \int_{M_{P}} \operatorname{Td}\left((T M)_{P}\right) \operatorname{Ch}\left(L_{P}\right)
$$

We emphazise that $(T M)_{P} \neq T M_{P}$, so that the terms on the right hand side of (32) do not equal $\operatorname{RR}\left(M_{P}, L_{P}\right)$.

Proof. Choose the connections on $T M$ and $L$ in such a way that for each $P \in \mathcal{P}$, the connection on $\left.T M\right|_{\psi^{-1}(\operatorname{int}(P))}$ and $\left.L\right|_{\psi^{-1}(\operatorname{int}(P))}$ equals the pull-back of a connection on the cut bundles $(T M)_{P}$ and $L_{P}$. Then the restriction of $\operatorname{Td}(M)$ and $\operatorname{Ch}(L)$ to $\psi^{-1}(\operatorname{int}(P))$ is the pull-back of $\operatorname{Td}\left((T M)_{P}\right)$ resp. $\operatorname{Ch}\left(L_{P}\right)$, so that (32) follows from the obvious identity

$$
\operatorname{RR}(M, L)=\sum_{P \in \mathcal{P}} \int_{\psi^{-1}(P)} \operatorname{Td}(M) \operatorname{Ch}(L)
$$

q.e.d.

A similar equation holds for $\operatorname{RR}\left(M_{Q}, L_{Q}\right)$. Note that $\left(M_{Q}\right)_{P}=$ $M_{Q \cap P}$ and, for any bundle $E \rightarrow M$ with lifts of the local torus actions, $\left(E_{Q}\right)_{P}=E_{Q \cap P}$. To prove the Theorem it suffices to show that for all $P$,

$$
\begin{aligned}
& \int_{M_{P}} \operatorname{Td}\left((T M)_{P}\right) \operatorname{Ch}\left(L_{P}\right) \\
& \quad=\sum_{Q \in \mathcal{Q}}(-1)^{\operatorname{codim} Q} \int_{M_{P \cap Q}} \operatorname{Td}\left(\left(T M_{Q}\right)_{P}\right) \operatorname{Ch}\left(L_{P \cap Q}\right) .
\end{aligned}
$$

More generally we prove the equivariant extension of this equation

$$
\begin{aligned}
\int_{M_{P}} \operatorname{Td}\left((T M)_{P}, \xi\right) \operatorname{Ch}\left(L_{P}, \xi\right) \\
=\sum_{Q \in \mathcal{Q}}(-1)^{\operatorname{codim} Q} \int_{M_{P \cap Q}} \operatorname{Td}\left(\left(T M_{Q}\right)_{P}, \xi\right) \operatorname{Ch}\left(L_{P \cap Q}, \xi\right)
\end{aligned}
$$

(for $\xi \in \mathfrak{t}_{(P)}$ small). To verify this Equation we apply the BerlineVergne localization formula to both sides and compare the $T_{(P)}$-fixed point contributions. 
Lemma A.8. For all $X \subset M_{P}^{T_{(P)}}$, the image $\psi_{P}(X)$ is contained in the interior of a unique polytope $Q \in \mathcal{Q}$ of maximal dimension.

Proof. Suppose $F$ is an open face of some polytope $Q \in \mathcal{Q}$ such that $F \cap \psi_{P}(X) \neq \emptyset$. Then $X$ is fixed under $T_{F} \subseteq T_{(P)}$, while according to (b) $T_{F}$ act locally freely on $X \cap \psi_{P}^{-1}(F)$. Thus $T_{F}=\{e\}$, showing that $\operatorname{dim} F=\operatorname{dim} T$. q.e.d.

Hence the fixed point contribution of $X \subset M_{P}$ appears exactly once as a fixed point contribution for a unique $Q$ on the right hand side.

Consider on the other hand a "new" fixed point manifold $X \subset M_{Q \cap P}^{T_{(P)}}$ for some $Q \in \mathcal{Q}$. Let $F$ be the largest open face of $Q$ containing $\psi(X)$ in its interior. Then $X$ is a fixed point manifold for all $M_{Q \cap P}$ such that $F$ is contained in $Q$ or equivalently $\psi(X) \subset Q$. The restriction $L_{X}:=L_{P \cap Q} \mid X$ does not depend on $Q$. The equality we wish to prove is

$$
\sum_{Q \supset \psi(X)}(-1)^{\operatorname{codim} Q} \int_{X} \frac{\operatorname{Td}\left(\left(T M_{Q}\right)_{P}, \xi\right) \operatorname{Ch}\left(L_{X}, \xi\right)}{\operatorname{Eul}\left(\nu_{X, Q \cap P}, \xi\right)}=0
$$

where $\nu_{X, Q \cap P}$ is the normal bundle of $X$ in $M_{Q \cap P}$. The bundle $\left(T M_{Q}\right)_{P}$ splits as $\left(T M_{Q}\right)_{P}=\left(T M_{F}\right)_{P} \oplus \nu_{F, Q}$ where $\nu_{F, Q}$ is the normal bundle of $\left(M_{F}\right)_{P}$ inside $\left(M_{Q}\right)_{P}$. On the other hand the normal bundle $\nu_{X, Q \cap P}$ splits as $\nu_{X, Q \cap P}=\left.\nu_{F, Q}\right|_{X} \oplus \nu_{X, F \cap P}$. Therefore

$$
\begin{gathered}
\operatorname{Td}\left(\left(T M_{Q}\right)_{P}, \xi\right)=\operatorname{Td}\left(\left(T M_{F}\right)_{P}, \xi\right) \operatorname{Td}\left(\nu_{F, Q}, \xi\right), \\
\operatorname{Eul}\left(\nu_{X, Q \cap P}, \xi\right)=\operatorname{Eul}\left(\nu_{X}, F \cap P, \xi\right) \iota_{X}^{*} \operatorname{Eul}\left(\nu_{F, Q}, \xi\right),
\end{gathered}
$$

and (35) follows from the local gluing rule [36]

$$
\sum_{Q \supset F}(-1)^{\operatorname{codim} Q} \frac{\operatorname{Td}\left(\nu_{F, Q}, \xi\right)}{\operatorname{Eul}\left(\nu_{F, Q}, \xi\right)}=0 .
$$

\section{References}

[1] R. Abraham, J. Marsden \& T. Ratiu, Manifolds, tensor analysis and applications, Addison-Wesley, Reading, 1983.

[2] S. Agnihotri \& C. Woodward, Eigenvalues of products of unitary matrices and quantum Schubert calculus, Technical report, Harvard, 1997, alg-geom/9712013. 
[3] M. F. Atiyah, The geometry and physics of knots, Cambridge Univ. Press, Cambridge, 1990.

[4] M. F. Atiyah \& R. Bott, The Yang-Mills equations over Riemann surfaces, Phil. Trans. Roy. Soc. London Ser. A 308 (1982) 523-615.

[5] M. F. Atiyah \& A. Pressley, Convexity and loop groups, Arithmetic and geometry, Progr. Math., Birkhäuser, Boston, Vol. II, No.36, 1983, 33-63.

[6] A. Beauville, Conformal blocks, fusion rules and the Verlinde formula, Proc. Hirzebruch 65 Conference on Algebraic Geometry (Ramat Gan, 1993), No. 9 in Israel Math. Conf. Proc., Bar-Ilan Univ., Ramat Gan, 1996, 75-96.

[7] A. Beauville \& Y. Laszlo. Conformal blocks and generalized theta functions, Comm. Math. Phys. 164 (1994) 385-419.

[8] N. Berline \& M. Vergne. Zéro d'un champ de vecteurs et classes caractéristiques équivariantes, Duke Math. J. 50 (1983) 539-549.

[9] J.M. Bismut \& F. Labourie. Formules de Verlinde pour les groupes simplement connexes et geometrie symplectique, C. R. Acad. Sci. Paris Ser. I Math. 325 (1997) 1009-1014.

[10] B. Booß-Bavnbek \& K. P. Wojciechowski, Elliptic boundary value problems for Dirac operators, Birkhäuser, Boston, 1993.

[11] R. Bott \& C. Taubes. On the rigidity theorems of Witten, J. Amer. Math. Soc. 2 (1989) 137-186.

[12] S. K. Donaldson, Boundary value problems for Yang-Mills fields, J. Geom. Phys. 8 (1992) 89-122.

[13] S. K. Donaldson \& P. Kronheimer, The geometry of four-manifolds, Oxford Math. Monographs. Oxford Univ. Press, New York, 1990.

[14] G. Faltings, A proof for the Verlinde formula, J. Differential Geom. 3 (1990) $347-374$

[15] H. Flaschka \& T. Ratiu, A convexity theorem for Poisson actions of compact Lie groups, Ann. Sci. École Norm. Sup. 29 (1996) 787-809.

[16] D. S. Freed, The geometry of loop groups, J. Differential Geom. 28 (1988) 223-276.

[17] V. Guillemin, Reduced phase spaces and Riemann-Roch, (R. Brylinski et al., eds.), Lie groups and geometry in Honor of B. Kostant, Progr. Math., Mass. Inst. Tech., Vol 123, 1994, 1995. Birkhäuser, Boston, 305-334.

[18] V. Guillemin \& S. Sternberg, Geometric quantization and multiplicities of group representations, Invent. Math. 67 (1982) 515-538.

[19] _._. The Gelfand-Cetlin system and quantization of the complex flag manifolds, J. Funct. Anal. 52 (1983) 106-128. 
[20] $\longrightarrow$, Symplectic techniques in physics, Cambridge Univ. Press, Cambridge, 1990.

[21] J. Huebschmann, Poisson structure on certain moduli spaces for bundles on a surface, Ann. Inst. Fourier (Grenoble) 45 (1995) 65-91.

[22] L. Jeffrey \& J. Weitsman, Toric structures on the moduli space of flat connections on a Riemann surface: volumes and the moment map, Adv. Math. 106 (1994) 151-168.

[23] L. C. Jeffrey, Extended moduli spaces of flat connections on Riemann surfaces, Math. Ann. 298 (1994) 667-692.

[24] L. C. Jeffrey \& F. C. Kirwan, Intersection pairings in moduli spaces of vector bundles of arbitrary rank over a Riemann surface, Preprint, McGill University, 1996. alg-geom/9608029.

[25] T. Kawasaki, The Riemann-Roch theorem for complex V-manifolds, Osaka J. Math. 16 (1979) 151-159.

[26] F. C. Kirwan, Cohomology of quotients in symplectic and algebraic geometry, Math. Notes, Vol. 31, Princeton Univ. Press, Princeton, 1984.

[27] , Partial desingularisations of quotients of nonsingular varieties and their Betti numbers, Ann. of Math. 122 (1985) 41-85.

[28] B. Kostant, Quantization and unitary representations, (C. T. Taam, ed.), Lectures in modern analysis and applications III, Lecture Notes in Math., Vol. 170, Washington, D.C., 1970. Springer, Berlin, 87-208.

[29] S. Kumar \& M.S. Narasimhan, Picard group of the moduli space of G-bundles, Math. Ann. 308 (1997) 155-173.

[30] S. Kumar, M.S. Narasimhan \& A. Ramanathan, Infinite Grassmannians and moduli spaces of G-bundles, Math. Ann. 300 (1994) 41-75.

[31] H. B. Lawson, Jr. \& M.-L. Michelson, Spin geometry, Princeton Math. Ser., Princeton Univ. Press, Princeton, 1989, Vol. 38.

[32] E. Lerman, Symplectic cut, Math. Res. Letters 2 (1995) 247-258.

[33] E. Lerman, E. Meinrenken, S. Tolman \& C. Woodward, Non-abelian convexity by symplectic cuts, Topology, 37 (1998) 245-259.

[34] V.B. Mehta \& T.R. Ramadas. Moduli of vector bundles, Frobenius splitting, and invariant theory, Ann. of Math. 144 (1996) 269-313.

[35] E. Meinrenken, On Riemann-Roch formulas for multiplicities, J. Amer. Math. Soc. 9 (1996) 373-389.

[36] Symplectic surgery and the Spin ${ }^{\mathrm{c}}$-Dirac operator, Adv. Math. 134 (1998) 240-277. 
[37] E. Meinrenken \& R. Sjamaar, Singular reduction and quantization, Topology, to appear. dg-ga/9707023.

[38] J. Mickelsson, String quantization on group manifolds and the holomorphic geometry of diff $S^{1} / S^{1}$, Comm. Math. Phys. 112 (1987) 653-661.

[39] A. Pressley \& G. Segal, Loop groups, Oxford Univ. Press, Oxford, 1988.

[40] T. R. Ramadas, I. Singer \& J. Weitsman, Some comments on Chern-Simons gauge theory, Comm. Math. Phys. 126 (1989) 409-420.

[41] G. Segal, Atiyah et al.: Seminar on Jones-Witten theory, Lecture notes, Oxford, 1988 .

[42] R. Sjamaar, Symplectic reduction and Riemann-Roch formulas for multiplicities, Bull. Amer. Math. Soc. (N.S.) 33 (1996) 327-338.

[43] R. Sjamaar \& E. Lerman, Stratified symplectic spaces and reduction, Ann. of Math. 134 (1991) 375-422.

[44] C. Sorger, La formule de Verlinde, Asterisque 237 (1996) 87-114; Bourbaki, Vol. 1994/95, Exp. No. 794.

[45] A. Szenes, The combinatorics of the Verlinde formulas, Vector bundles in algebraic geometry (Durham, 1993), London Math. Soc. Lecture Note Ser., No. 208, Cambridge Univ. Press, 1995, 241-253.

[46] C. Teleman, Lie algebra cohomology and the fusion rules, Comm. Math. Phys. 173 (1995) 265-311.

[47] - Verlinde factorization and Lie algebra cohomology, Invent. Math. 126 (1996) 249-263.

[48] A. Tsuchiya, K. Ueno \& Y. Yamada, Conformal field theory on universal family of stable curves with gauge symmetries, Integrable systems in quantum field theory and statistical mechanics, Adv. Stud. Pure Math., Vol. 19, Academic Press, 1989, $459-566$.

[49] M. Vergne, Quantification géométrique et multiplicités, C. R. Acad. Sci. Paris Sér. I Math. 319 (1994) 327-332.

[50] __ Equivariant index formula for orbifolds, Duke Math. J. 82 (1996) 637-652.

[51] E. Verlinde, Fusion rules and modular transformations in $2 d$ conformal field theory, Nuclear Phys. B 300 (1988) 360-376.

[52] E. Witten, Two-dimensional gauge theories revisited, J. Geom. Phys. 9 (1992) 303-368. 
